\title{
The Application of High Pressure in Physics and Technology of III-V Nitrides
}

I. Grzegory, S. Krukowski, M. Leszczyński, P. Perlin, T. Suski AND S. Porowski

High Pressure Research Center, Polish Academy of Sciences, Unipress

Sokołowska 29/37, 01-142 Warsaw, Poland

Due to high bonding energy of $\mathrm{N}_{2}$ molecule, the III-V semiconducting nitrides, especially GaN and $\mathrm{InN}$ require high $\mathrm{N}_{2}$ pressure to be stable at high temperatures necessary for growth of high quality single crystals. Physical properties of $\mathrm{GaN}-\mathrm{Ga}(\mathrm{l})-\mathrm{N}_{2}$ system are discussed in the paper. On the basis of the experimental equilibrium $p-T-x$ data and the quantum-mechanical modeling of interaction of $\mathrm{N}_{2}$ molecule with liquid Ga surface, the conditions for crystallization of GaN were established. The crystals obtained under high pressure are of the best structural quality, having dislocation density as low as $10-100 \mathrm{~cm}^{-2}$ which is several orders of magnitude better than in any other crystals of GaN. The method allows to grow both $n$-type substrate crystals for optoelectronics and highly resistive crystals for electronic applications. The physical properties of the pressure grown GaN measured to characterize both point defects and extended defects in the crystal lattice are discussed in the paper. A special attention is paid to the application of high pressure to reveal the nature of the point defects in the crystals and electric fields in GaN-based quantum structures. Due to their very high structural quality, the pressure grown crystals are excellent substrates for epitaxial growth of quantum structures. It opens new possibilities for optoelectronic devices, especially short wavelength high power lasers and efficient UV light emitting diodes. This is due to the strong reduction in dislocation densities in relation to existing structures $\left(10^{6}-10^{8} \mathrm{~cm}^{-2}\right)$ which are grown on strongly mismatched sapphire and $\mathrm{SiC}$ substrates. The experimental results on the epitaxial growth and physical properties of GaN-based device structures supporting above conclusions are discussed in the paper. The current development of blue laser technology in High Pressure Research Center is shortly reviewed.

PACS numbers: 81.10.-h, 78.66.Fd, 73.61.Ey, 82.60.Lf, 78.55.Cr 


\section{Introduction}

GaN and its alloys with $\mathrm{Al}$ and In became recently the basic materials for short-wavelength optoelectronics. It was mainly due to their direct energy gaps covering the whole visible spectrum and the large part of the UV range $(6.2,3.4$, and $1.9 \mathrm{eV}$ for $\mathrm{AlN}, \mathrm{GaN}$, and $\mathrm{InN}$, respectively). These materials besides of being a prime object of interest of device engineers are also exciting subject of research for a physicist. This is because of their outstanding position in III-V family of semiconductors. GaN, InN, and AlN unlike other III-V materials are hard, very ionic semiconductors of high chemical and thermal stability. From the point of view of ionicity they rather resemble II-VI compound like ZnS, from the point of view of their hardness and chemical inertness they rather appear to be similar to diamond or boron nitride.

Although GaN was first synthesized long time ago (in early thirties) the development of this group of semiconductors was hampered by extreme difficulties of growing them in single crystalline form. This latter fact span from the thermodynamic properties of e.g. GaN which has a melting temperature very high $2500^{\circ} \mathrm{C}$ - which is accompanied by high equilibrium nitrogen pressure close to 45000 bar. Between roughly 1965-1975 Jacques Pankove and his students did a pioneering work in growing and characterizing gallium nitride. Pankove himself, was even at this time, aware of importance of this material in yet to exist new branch of electronics - optoelectronics. In the middle of seventies Pankove had to give up nitride research, facing problems too difficult for the technology of these times.

The comeback of nitrides occurred in 1989 thanks to Japanese scientists Amano, Akasaki, and Nakamura who overcame the problems of growth and $p$-type doping. They used modern metal-organic chemical vapor deposition (MOCVD) technique to grow nitride $(\mathrm{GaN}$, InGaN) layers on sapphire substrates. They used also a post growth activation process to get a $p$-type material. From this time on the development of this materials is biased by the industrial demand for short-wavelength light emitting diodes and lasers.

At present, high brightness blue and green light emitting diodes (LEDs) and low power blue laser diodes (LDs) are commercially available [1]. On the other hand, however, the development of GaN-based technology was and still is, strongly limited by difficulties in obtaining large, high quality GaN crystals which could be used as substrates for epitaxial deposition of multilayer quantum structures necessary for devices. This is a direct consequence of thermodynamic properties of GaN [2] (and also AlN [3]), in particular, its melting conditions which are so extreme that the application of the common growth methods from stoichiometric liquids is technically impossible. The only method of growing bulk crystals of nitrides successful so far is the growth of $\mathrm{GaN}$ under high pressure of nitrogen. High Pressure Research Center Unipress has been leading the research on bulk gallium nitride growth for almost twenty years. In this paper we would like to give a reader 
a summary of multidirectional activity of High Pressure Research Center (HPRC) in the field of nitride science. In particular, we will address the problem of bulk GaN crystal growth and their use as substrates for MOCVD epitaxial deposition. In parallel we would like to draw the reader's attention to use of high-pressure techniques in the studies of physical properties of these materials. We hope to demonstrate that frequently high-pressure techniques are really enabling tools in solving very fundamental problems related to physics of these materials.

\section{High pressure crystallization of GaN}

\subsection{Thermodynamic properties of $\mathrm{GaN}-\mathrm{Ga}-\mathrm{N}_{2}$ system}

In Table the melting temperatures and pressures of most typical semiconductor materials are compared. The melting temperature $T^{\mathrm{M}}$, and the corresponding equilibrium pressure at melting, of GaN, AlN, and $\mathrm{InN}$ has not been measured. The quoted melting temperatures has been calculated by the use of Van Vechten's quantum dielectric theory of chemical bonding [4]. The corresponding pressure follows from the extrapolation of the experimental equilibrium data [5, 6]. Table shows that both temperature and pressure at melting of GaN are much higher than that for typical semiconductors. They are rather similar to the conditions used for high-pressure synthesis of diamond.

\section{TABLE}

Melting conditions of semiconductors.

\begin{tabular}{c|c|c}
\hline \hline Crystal & $T^{\mathrm{M}}\left[{ }^{\circ} \mathrm{C}\right]$ & $p^{\mathrm{M}}[\mathrm{atm}]$ \\
\hline $\mathrm{Si}$ & 1400 & $<1$ \\
GaAs & 1250 & 15 \\
$\mathrm{GaP}$ & 1465 & 30 \\
$\mathrm{AlN}$ & 3200 & 200 \\
$\mathrm{GaN}$ & $\mathbf{2 5 0 0}$ & $\mathbf{4 5 0 0 0}$ \\
$\mathrm{InN}$ & 1900 & $>60000$ \\
\hline diamond & & \\
(synthesis) & 1600 & 60000
\end{tabular}

Due to these extreme melting conditions, GaN (like AIN [3] and $\operatorname{InN}$ [7]) cannot be grown from its stoichiometric melt by the Czochralski or Bridgman methods commonly used for typical semiconductors. It has to be crystallized by methods allowing to grow the bulk crystals using lower temperatures and pressures.

Gallium nitride is strongly bonded compound (with bonding energy of $9.12 \mathrm{eV} /$ atom pair [8]) in comparison with typical III-V semiconductors like GaAs 
(bonding energy of $6.5 \mathrm{eV} /$ atom pair). Consequently, the free energy of the crystal is very low in relation to the reference state of free $\mathrm{N}$ and Ga atoms. On the other hand, the $\mathrm{N}_{2}$ molecule is also strongly bonded $(4.9 \mathrm{eV} /$ atom $)$. Therefore, the free energy of GaN constituents at their normal states, $\mathrm{Ga}$ and $\mathrm{N}_{2}$, becomes quite close to that of the crystal. It is illustrated in Fig. 1 where the free energy of $\mathrm{GaN}$ ( 1 mole) and the free energy of the system of its constituents $\left(\mathrm{Ga}+(1 / 2) \mathrm{N}_{2}\right)$ is shown as a function of temperature and $\mathrm{N}_{2}$ pressure.

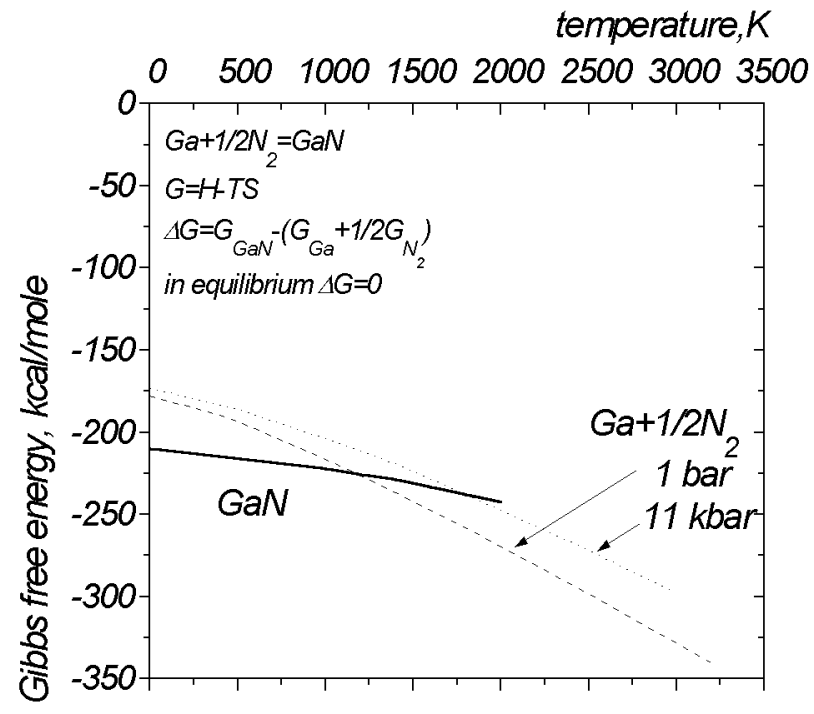

Fig. 1. Gibbs free energy of GaN and its constituents.

With increasing temperature, Gibbs composite free energy of the constituents $G(T)$ decreases faster than $G(T)$ of the crystal and at higher temperatures, the nitride becomes thermodynamically unstable. The crossing of $G(T)$ curves determines the equilibrium temperature where GaN coexists with its constituents at given $\mathrm{N}_{2}$ pressure. The application of pressure increases the free energy of the constituents in much higher degree than $G(T)$ of the crystal. As a consequence, the equilibrium point shifts to higher temperatures and GaN stability range extends. The equilibrium $p_{\mathrm{N}_{2}}-T$ conditions for $\mathrm{GaN}$ have been studied by several groups $[7,9,10]$. The most complete and consistent results have been obtained by Karpinski et al. [5, 6] by direct synthesis and decomposition experiments performed by both gas pressure technique (for pressures up to $20 \mathrm{kbar}$ ) and high pressure anvil technique (up to $70 \mathrm{kbar}$ ). The curve following from these data is shown in Fig. 2. Crystallization processes discussed in this paper have been carried out at $\mathrm{N}_{2}$ pressure up to 20 kbar which corresponds to GaN stability limit of $1960 \mathrm{~K}$. These conditions are marked in Fig. 2. As it was shown in Ref. [6] for pressures up to $20 \mathrm{kbar}$, the equilibrium curve can be described by the Van't Hoff equation 


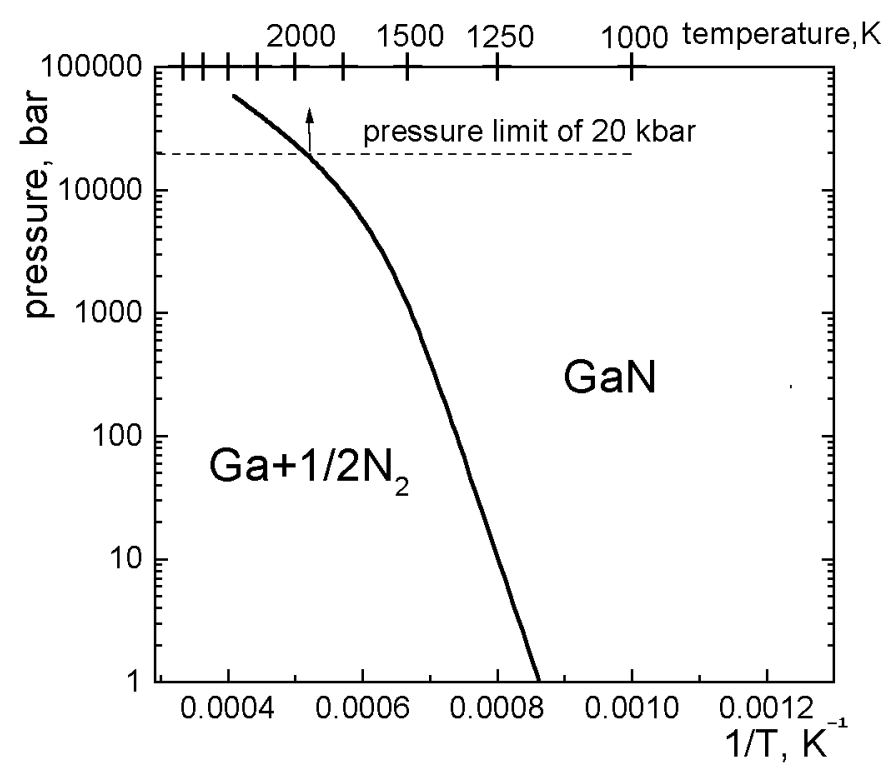

Fig. 2. Equilibrium curve for GaN [5, 6].

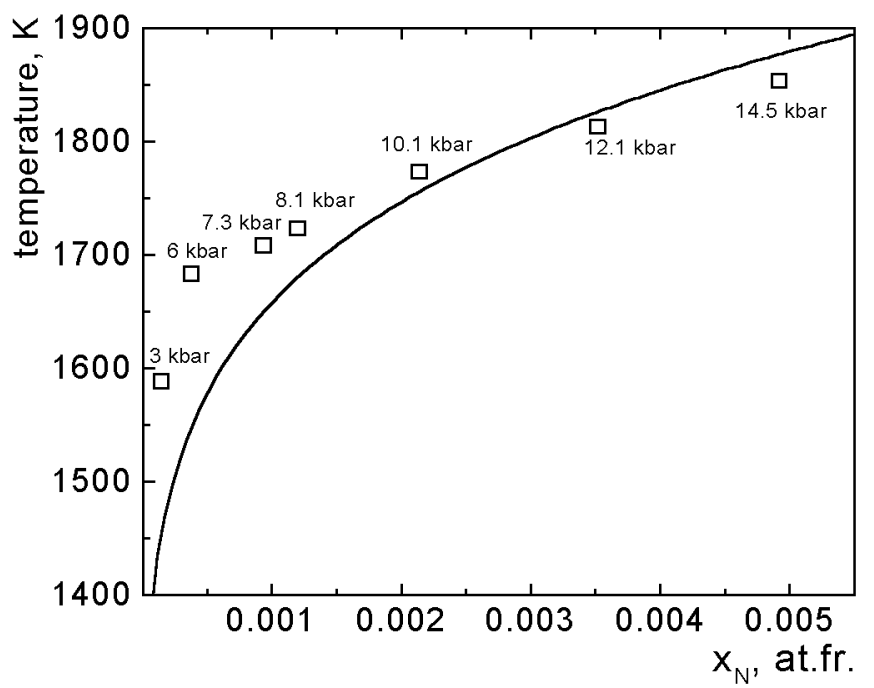

Fig. 3. Liquidus line for Ga-GaN system: the solid line was calculated in the ideal solution approximation.

$$
\Delta H_{\mathrm{F}}=\frac{1}{2} R \frac{\mathrm{d} \ln a_{\mathrm{N}_{2}}}{\mathrm{~d}(1 / T)},
$$

where $\Delta H_{\mathrm{F}}$ is the formation enthalpy of $\mathrm{GaN}, a_{\mathrm{N}_{2}}$ is the equilibrium activity of $\mathrm{N}_{2}$ gas, with $\Delta H_{\mathrm{F}}$ constant and equal to $-37.7 \mathrm{kcal} /$ mole. The extension of GaN stability range by the application of pressure allows to grow GaN crystals from the solution in the liquid Ga. In Fig. 3 we have shown the experimental N solubility 
data [8] resulting from the annealing of $\mathrm{Ga}$ in $\mathrm{N}_{2}$ atmosphere at the three phase equilibrium conditions. Even the highest experimentally accessible temperature $1960 \mathrm{~K}$ is quite far from the melting temperature of GaN (Table). Therefore the $\mathrm{N}$ concentrations are not high (below 1 at.\%) and the growth experiments have to be long to get high quality crystals with dimensions appropriate for research and applications. Therefore, the long time $(>100 \mathrm{~h}$ ) of stable work is an additional requirement for the growth system.

The solid line in Fig. 3 is the liquidus line for $\mathrm{Ga}-\mathrm{GaN}$ system calculated in ideal solution approximation with Van Vechten's melting temperature of $2790 \mathrm{~K}$ [4]. For this approximation the solubility can be expressed as follows:

$$
n=n_{0} \exp \frac{\Delta H_{\mathrm{sol}}}{k T}
$$

where $\Delta H_{\text {sol }}$ is the heat of dissolution. For GaN, $\Delta H_{\text {sol }}=44.7 \mathrm{kcal} / \mathrm{mole}=$ $0.49 \mathrm{eV} /$ bond and expresses the bonding energy in the crystal in relation to its mother phase - the solution [8].

The analysis of thermodynamic properties of the system of $\mathrm{GaN}$ and its constituents explains the role of high pressure as a factor increasing thermodynamic potential of the constituents which makes the crystal stable at temperatures necessary for crystallization. The pressure however is important also for kinetics of $\mathrm{GaN}$ synthesis. It is analyzed in the next section.

\subsection{Dissolution kinetics and crystal growth mechanism}

The nature of $\mathrm{GaN}-\mathrm{Ga}(\mathrm{l})-\mathrm{N}_{2}$ system plays important role not only in the thermodynamics of this system. The kinetic properties are also much different from other III-V systems which entails the use of specific experimental crystal growth techniques. This also affects the models used in the description of the microscopic properties of the system and simulation procedures.

Although the physical properties of these three phases are completely different, ranging from the gas $\left(\mathrm{N}_{2}\right)$ to metallic liquid $(\mathrm{Ga})$ and semiconductor solid $(\mathrm{GaN})$, they have some common features. The most important is the fact that the atoms in all three phases are very strongly bound. Molecular nitrogen is the strongest bonded diatomic molecule in the nature, the dissociation energy of $\mathrm{N}_{2}$ molecule is $9.76 \mathrm{eV}$ [8]. Gallium liquid is characterized by high enthalpy of evaporation $271 \mathrm{~kJ} / \mathrm{mole}(2.81 \mathrm{eV} /$ atom $)$ which is reflected by the critical temperature, close to $4000 \mathrm{~K}[11,12]$. Similarly, solid $\mathrm{GaN}$ is strongly bound, with the energy $9.12 \mathrm{eV} /$ atom pair, which leads to high melting temperature, that according to Van Vechten estimate is close to $2790 \mathrm{~K}$ [4].

GaN synthesis from its constituents proceeds via dissolution of nitrogen in liquid gallium, transport of nitrogen to the cold part of the liquid and the growth from the solution. These stages include dissociation of $\mathrm{N}_{2}$ molecule which is energetically costly process. It is therefore expected that the dissociation process 
involves significant change of the binding energy of the molecule, highly exceeding the typical thermal motion energies, which are of order of $0.15 \mathrm{eV}$.

It is likely that the dissociation occurs during the adsorption of the $\mathrm{N}_{2}$ molecules on liquid Ga surface. This possibility was investigated solving quantum mechanical many-body problem using density functional method (DFT) formulation [13]. Since thermal motion of the Ga atoms plays minor role, the BornOppenheimer approximation can be used. An infinite Ga surface can be simulated by finite cluster of Ga atoms $[14,15]$.
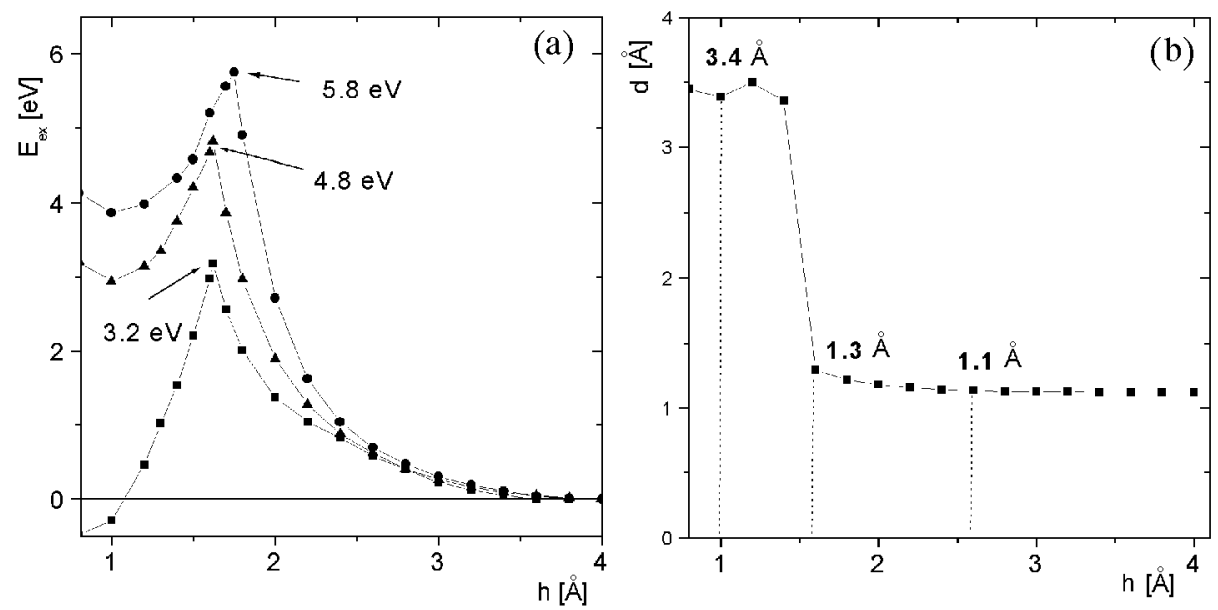

Fig. 4. (a) Excess energy of the system in function of the distance between the $\mathrm{N}_{2}$ molecule and metal cluster: squares - Al, triangles - Ga, circles - In. (b) N-N distance in function of the distance between the $\mathrm{N}_{2}$ molecule and Ga cluster.

The two different orientations of $\mathrm{N}_{2}$ molecule: parallel and perpendicular to the surface were used in the calculations [14]. The obtained results include the electron charge distribution, the position of the atoms, and the total energies of the system. In Fig. $4 \mathrm{a}$ we present the change of the total energy of the system as a function of the distance between $\mathrm{Ga}$ surface and $\mathrm{N}_{2}$ molecule. In these data the parallel orientation of the molecule to the surface has been used.

The interaction is negligible for distances higher than $4 \AA$ for which the excess energy zero level is adopted. For closer distances the energy increases sharply to reach $4.8 \mathrm{eV}$ for $d=1.6 \AA$ and then sharply decreases. As shown in Fig. 4b, the excess energy increase occurs when the $\mathrm{N}-\mathrm{N}$ distance is only slightly increased whereas the decrease occurs after the $\mathrm{N}-\mathrm{N}$ distance increased suddenly to more than $3.2 \AA$. This dramatic change indicates that at the distance $1.6 \AA$, the dissociation of $\mathrm{N}_{2}$ molecule takes place.

The dissociation of $\mathrm{N}_{2}$ molecule is confirmed by the plot of the electronic charge distribution in Fig. 5. The constant charge surface was plotted for the three selected $\mathrm{Ga}-\mathrm{N}_{2}$ distances: $4.0 \AA, 1.6 \AA$, and $1.0 \AA$. For the first two cases 


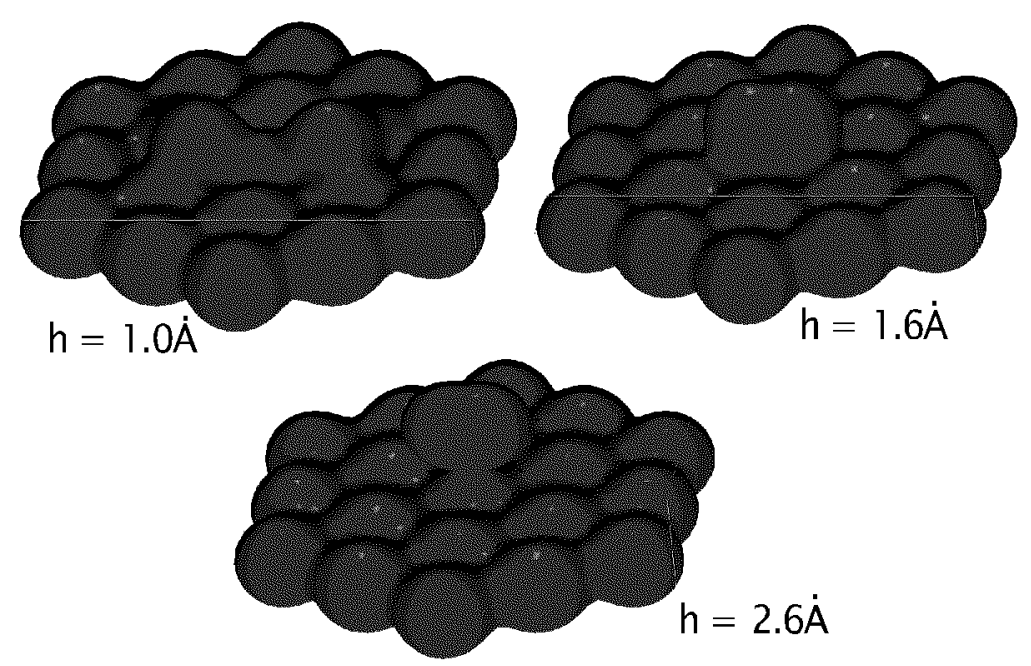

Fig. 5. Electron constant density surface for $\mathrm{N}_{2}$, parallel to 19 atoms Ga cluster for three selected N-Ga distances: $h=2.6 \AA, h=1.6 \AA$, and $h=1.0 \AA$.

the electronic charge is concentrated in the region between $\mathrm{N}$ atoms, for the last case the charge is shifted to the region between $\mathrm{Ga}$ and $\mathrm{N}$ atoms, confirming dissociation of $\mathrm{N}_{2}$ molecule and creation of chemical bond between two $\mathrm{N}$ atoms and Ga surface atoms.

The same behavior is observed for other group-III metals: Al and In. The energy barriers are: $3.2 \mathrm{eV}$ for $\mathrm{Al}$ and $5.8 \mathrm{eV}$ for $\mathrm{In}$, which are much lower than $\mathrm{N}_{2}$ dissociation energy, indicating on strong catalytic influence of group-III metal surface.

The calculation of the interaction of $\mathrm{N}_{2}$ molecule, oriented perpendicular to Ga surface also confirmed the dissociation of the molecule. Generally, the energy barriers are much lower for this orientation: $3.0 \mathrm{eV}$ for $\mathrm{Al}, 3.4 \mathrm{eV}$ for $\mathrm{Ga}$ and $3.6 \mathrm{eV}$ for In. Since the interatomic distances are much larger for In cluster, one can expect that the energy barrier for $\mathrm{Al}$ and $\mathrm{Ga}$ are relatively overestimated due to larger stiffness of these two clusters.

Using these energy barriers, the nitrogen dissociation rate on the metal surfaces was calculated. In these calculations the vibrational energy was accounted for. Since the energy barrier is much higher for the parallel than for perpendicular $\mathrm{N}_{2}$ orientation, the rotational energy was neglected. The impingement rate was obtained from ideal gas approximation. The reaction rate was determined as the fraction of the molecule which can penetrate over the energy barrier determined from the quantum-mechanical (QM) calculations. The temperature dependence of the nitrogen dissociation reaction rate for $\mathrm{Ga}, \mathrm{Al}$, and surfaces, for the gas pressure 20 kbar is presented in Fig. 6.

The nitrogen dissolved in the hot end Ga surface is transported into the cold part of the liquid by convection and diffusion. There the GaN growth proceeds 


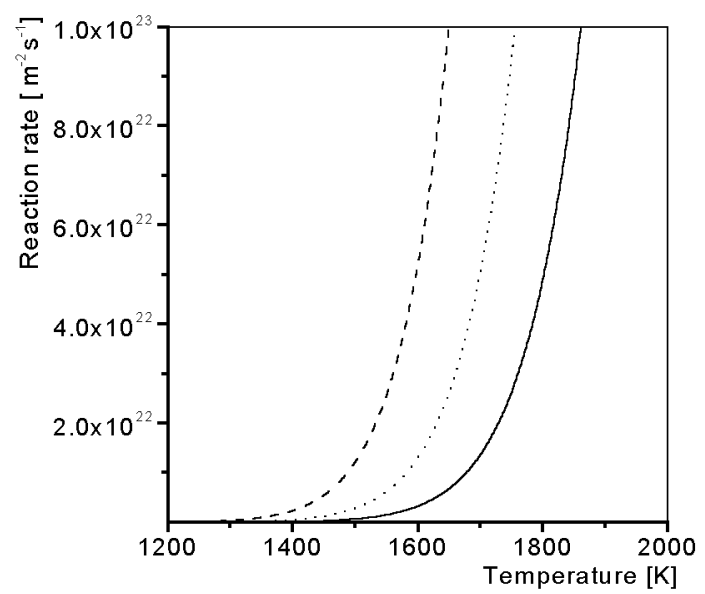

Fig. 6. Average dissociation rate of $\mathrm{N}_{2}$ on liquid group-III metal surface calculated using ideal gas approximation; $\mathrm{Al}$ - dashed line, Ga - dotted line, In - solid line.

via three main stages. The initial stage is heterogeneous nucleation of GaN on Ga surface. During second stage the growth of numerous GaN crystals and separation of the dominant growth centers occurs. And final stage is the growth of small number of single crystals in the supersaturated solution of $\mathrm{N}$ in liquid Ga. The last stage determines the results of the crystallization process.

Due to high bonding energy and crystallographic structure of main surfaces, growth of GaN crystals is strongly anisotropic. This is reflected in the shape of GaN crystals which is hexagonal platelet. From that observation follows that the growth rates are the fastest for $\{10 \underline{11}\}$ and $\{10 \underline{11}\}$, slower for $\{10 \underline{10}\}$ and the slowest for $\{0001\}$ and $\{000 \underline{1}\}$ surfaces. The flat faces of GaN crystals indicate that they grow by layer mechanism. Depending on the structure of the crystals and thermodynamic conditions at the interface several growth regimes can be distinguished.

One of the most efficient growth centers are screw dislocations. Since in GaN wurtzite structure screw dislocations have much higher energies than edge dislocation, their fraction in total dislocation density is small. In case of the growth of good quality GaN crystals, the role of screw dislocation is negligible.

In absence of the screw dislocation the morphological properties of GaN growth mode result from the competition between the nucleation and completion of new layers. These two processes have different size dependence. At stable thermodynamic conditions at the surface the nucleation rate is proportional to the surface whereas the completion rate is inversely proportional to the dominant linear size of the surface. In most cases it is proportional to the inverse of the square root of the surface. Hence one can expect that for some cases the transition from the nucleation to completion controlled growth occurs.

Growth controlled by $2 \mathrm{D}$ nucleation is morphologically stable. In most cases the surfaces (10-10) and (000-1) are atomically flat with several steps. The growth 
rate can be assessed using the Becker-Doring nucleation law. The rate is strongly dependent on supersaturation at the surface. Assuming that the edge energy is due to 2 broken bonds per atom site and using dissolution energy as the broken bond energy difference, the rate can be expressed as a function of the supersaturation [8]. For experimental growth rates, these calculations give $48 \%$ supersaturation at the growth zone, which is in good agreement with the estimation obtained from the temperature difference in the crucible and the $x-T$ phase diagram [8].

For other GaN faces the surfaces are not so flat. This is typical of the growth controlled by step flow where nucleation rate is so fast that allows to obtain numerous $2 \mathrm{D}$ nuclei at various parts of the surface before the layer is completed. This can be caused either by the step pinning due to the kink poisoning by the impurities or by extremely fast nucleation resulting from relatively high supersaturation. Also the presence of screw dislocation growth centers can cause similar effect. As a result, growth is much faster in some part of the surface leading to the creation of hill and valley morphology and the transition to the morphologically unstable forms. This type of the growth is observed sometimes in the growth on $\{0001\}$ surfaces.

\subsection{What happens with GaN at high temperature if the pressure is too low?}

Both the increase in thermodynamic potential of gaseous nitrogen and the enhancement of the creation of the atomic nitrogen at the Ga surface can be achieved by other (not compression) methods like the excitement of $\mathrm{N}_{2}$ plasma or the use of species containing nitrogen atoms bonded weaker than in $\mathrm{N}_{2}$ molecule. It was demonstrated [8] that in microwave nitrogen plasma of 60 Torr and temperature as low as $1100^{\circ} \mathrm{C}$, a very efficient $\mathrm{GaN}$ (and even $\mathrm{InN}$ !) synthesis is possible. The excited $\mathrm{N}_{2}$ gas was very efficient source to saturate a $\mathrm{Ga}$ droplet with atomic nitrogen and to form GaN crust on the droplet surface. However, since the pressure in the system was much lower than the equilibrium one (about 100 bar for $1100^{\circ} \mathrm{C}$ ), the $\mathrm{N}_{2}$ gas phase started to nucleate and grow in the liquid. This resulted in the formation of the structure shown in Fig. 7 which was the empty "Ga droplet" covered with irregular polycrystalline GaN.

The formation of the $\mathrm{N}_{2}$ bubbles in the supersaturated Ga: $\mathrm{N}$ liquid was also observed if the pressure in the system containing GaN crystals dipped in the Ga:N solution was intentionally decreased below the equilibrium value. Then the $\mathrm{N}_{2}$ bubbles nucleated on the surface of the crystals and the crystals being locally in contact with the gas started to decompose trying to restore the equilibrium. During cooling of the system, the interface between the gas bubbles and the surrounding liquid covered with GaN crystallized from the supersaturated solution. Two examples of GaN crystals with local decomposition features on their surfaces are shown in Fig. 8 together with the schematic illustration of the described process. 


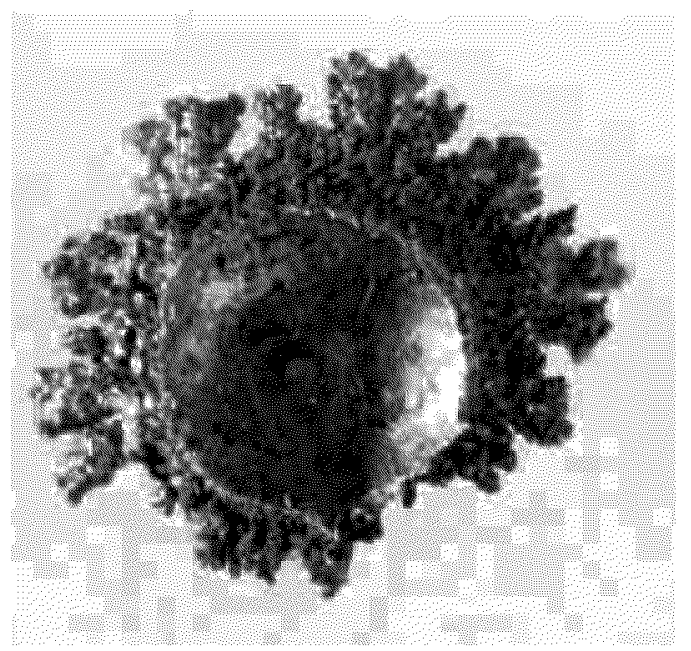

Fig. 7. The result of GaN synthesis from the liquid $\mathrm{Ga}$ and $\mathrm{N}_{2}$ plasma. The liquid has been pushed out from the initial droplet covered by GaN crust, by the growing $\mathrm{N}_{2}$ bubbles.

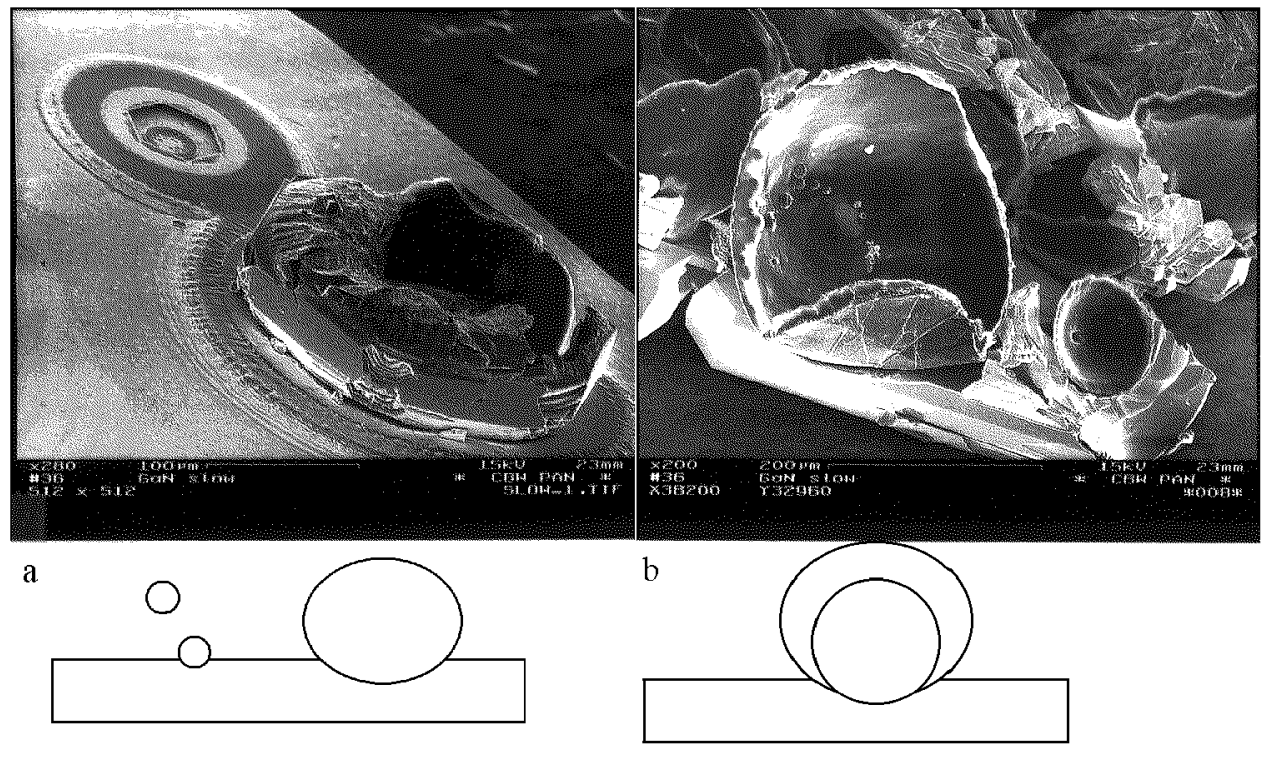

Fig. 8. Decomposition of GaN in the supersaturated Ga: $\mathrm{N}$ solution if the pressure in the system drops below the equilibrium value. Local decomposition features supporting the proposed mechanism are visible on the crystal surfaces. (a) $\mathrm{N}_{2}$ bubbles start to nucleate in the liquid, some of them nucleate on the GaN crystal surfaces, (b) during cooling the interfaces cover with GaN since the solution is supersaturated. 


\subsection{Crystallization of $G a N$ by high nitrogen pressure solution growth (HNPSG) method - experimental}

At present, GaN is crystallized in gas pressure chambers with volume up to $1500 \mathrm{~cm}^{3}$ allowing crucibles with the working volume of $50-100 \mathrm{~cm}^{3}$. The high pressure-high temperature reactor consisting of the pressure chamber and the multizone furnace is equipped with additional systems necessary for: in situ annealing in vacuum, electronic stabilization and programming of pressure and temperature, cooling of the pressure chamber. Pressure in the chamber is stabilized with the precision better than 10 bar. The temperature is measured by a set of thermocouples arranged along the furnace and coupled with the standard input power control electronic systems based on Eurotherm units. This allows stabilization of temperature $\pm 0.2 \mathrm{deg}$ and programmable changes of temperature distribution in the crucible. The example of high pressure apparatus, constructed in HPRC is presented in Fig. 9.

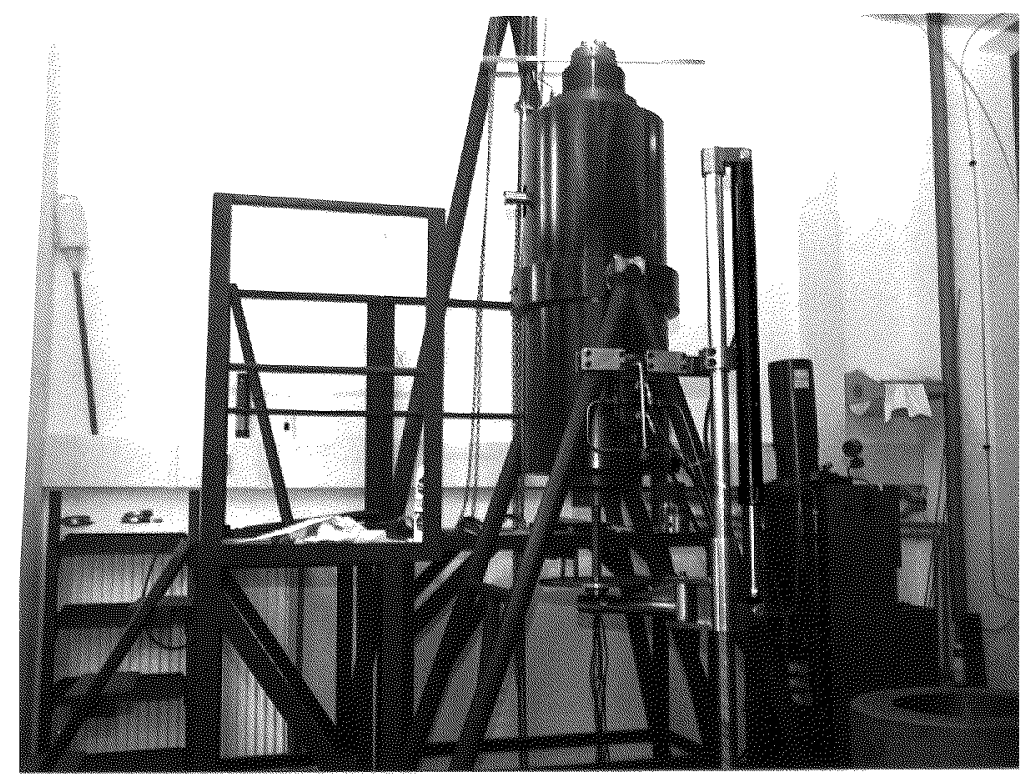

Fig. 9. High pressure apparatus, constructed in HPRC for crystallization of GaN. The maximum working pressure is $15 \mathrm{kbar}$, the maximum temperature $-1600^{\circ} \mathrm{C}$, the internal diameter $-100 \mathrm{~mm}$.

GaN crystals presented in this paper were grown from the solutions in pure liquid gallium and in Ga alloyed with $0.2-0.5$ at.\% of $\mathrm{Mg}$ or Be at pressures in the range of $10-20 \mathrm{kbar}$ and temperatures of $1400-1600^{\circ} \mathrm{C}$. Magnesium and beryllium as the most efficient acceptors in GaN were added to the growth solutions in order to reduce the concentration of free electrons in the crystals by compensation of residual donors. 
The supersaturation in the growth solution has been created by the application of temperature gradient of $2-20^{\circ} \mathrm{C} / \mathrm{cm}$ along the axis of the crucible. This method was chosen since the axial temperature gradients in multizone furnaces working at high gas pressure can be controlled with high precision and the method assures a continuous flow of nitrogen from the hotter part of the solution to the cooler one. If the crystallization experiments were performed without an intentional seeding, the crystals nucleated spontaneously on the internal surface of polycrystalline GaN crust covering liquid Ga at the cooler zone of the solution. Typical duration of the processes was 120-150 hours.

The slow cooling at isothermal conditions was not applied due to small concentrations of nitrogen in the liquid gallium (Fig. 3). The crystallization at constant temperature, at $\mathrm{N}_{2}$ overpressure was not applied as well, since then the crystallization can occur only on the Ga surface and stops if the whole surface covers with GaN.

\subsection{GaN single crystals}

\subsubsection{Crystals grown without intentional seeding}

The GaN crystals grown by the high nitrogen pressure solution method are of wurtzite structure, mainly in the form of hexagonal platelets. The structure of GaN crystal is presented in Fig. 10. As shown in the figure, the subsequent planes

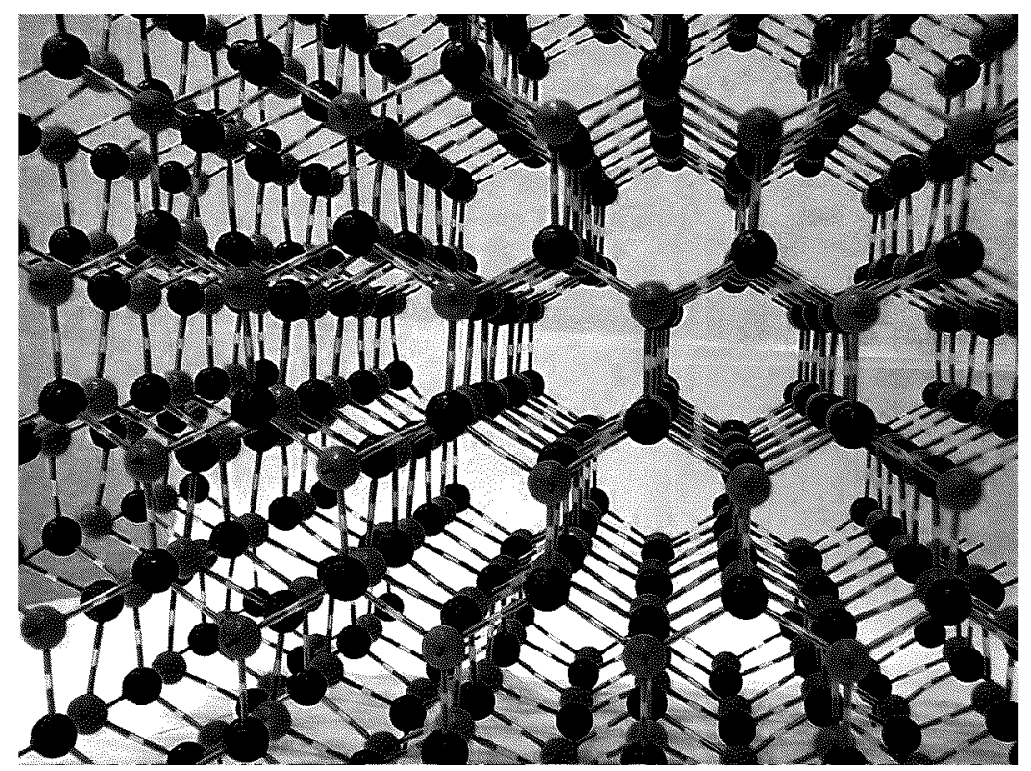

Fig. 10. The crystallographic structure of GaN crystals. Ga and N atoms are denoted by black and yellow color. 
consist of the atoms of the same kind. In one of these two planes, the atoms are bonded to three atoms. From the upper side these atoms are denoted as black, in the lower by yellow. These planes are energetically stable, therefore the sides are denoted as N-side and Ga-side.

The large hexagonal surfaces of bulk GaN crystals correspond to $\{0001\}$ polar crystallographic planes. Conventionally, the N-side is denoted as $\{000-1\}$ and Ga-side as $\{0001\}$ surface. The side faces of the crystals are mainly the polar $\{10-11\}$ and also non-polar $\{10-10\}$ planes.

The crystals in the form of hexagonal platelets grown slowly, with a rate below $0.1 \mathrm{~mm} / \mathrm{h}$ into $\{10 \underline{10}\}$ directions (perpendicular to the $c$-axis), are usually single crystals of perfect morphology suggesting stable layer-by-layer growth. They are transparent, with flat mirror-like faces. The habit of the crystals does not change for solutions containing $\mathrm{Mg}$ or $\mathrm{Be}$. The average size of crystals grown without an intentional seeding scales with the diameter of the high pressure reactor as it is shown in Fig. 11.
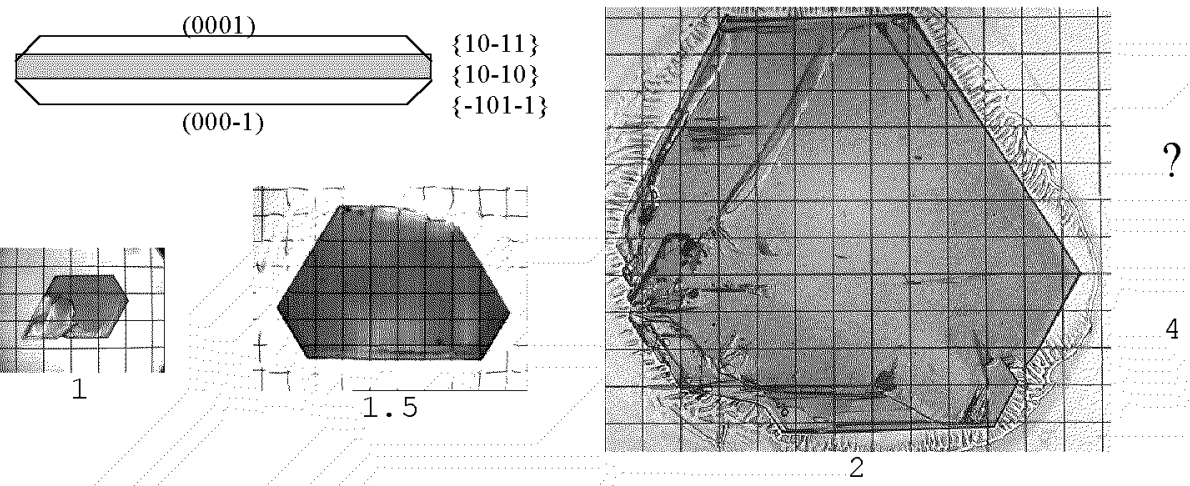

Fig. 11. GaN crystals grown at high pressure chambers of different sizes. The numbers are proportional to the diameters of the chambers. The distances between grid lines correspond to $1 \mathrm{~mm}$. The schematic cross-section of the hexagonal platelet is shown at the left top of the figure.

As one can deduce from the form of the crystals the growth is strongly anisotropic being much faster (about 100 times) in directions perpendicular to $c$-axis. This relation is valid at supersaturations corresponding to the average growth rate in $\{10 \underline{10}\}$ directions of $0.05-0.1 \mathrm{~mm} / \mathrm{h}$.

High supersaturations enhance the growth into $c$-direction which leads to the unstable needle-like forms. The supersaturation in the growth solution is determined mainly by the growth temperature, temperature gradients, mass transport mechanisms in gallium and also by the local surrounding for a particular crystal (i.e. the presence of neighboring crystals). For crystallization of large GaN crystals, it is crucial to control the supersaturation in order to avoid acceleration of 
the growth near edges and corners of the growing crystal. If supersaturation is too high, the edge nucleation on hexagonal faces of GaN platelets is often observed, which is the first step to the unstable growth on that faces. The result of such a growth is shown in Fig. 12a.
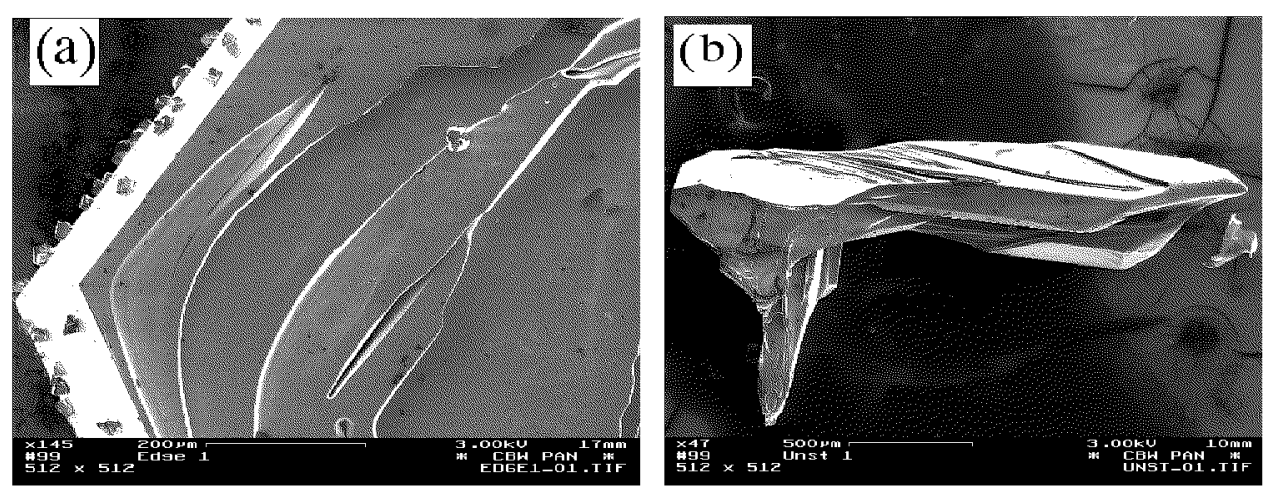

Fig. 12. (a) Edge nucleation on (0001) face of pressure grown GaN crystal. (b) Hollow GaN crystal with (1010) face formed at increased supersaturation.

In the extreme cases of very high supersaturations, the growth into $c$-direction nucleated at the edges of the plate, becomes very fast which leads to the formation of well developed $\{10 \underline{10}\}$ faces. Since the lateral growth on the $c$-face is still slow, the resulting crystals are hollow needles elongated into the $c$-directions (Fig. 12b).

The tendency for the unstable growth is stronger for one of the polar $\{0001\}$ faces of the platelets. On this side the morphological features like macrosteps, periodic inclusions of solvent, or cellular growth structures are observed. The opposite surface is always mirror-like and often atomically flat. For crystals grown without an intentional doping (strongly $n$-type - see next section) the unstable surface always corresponds to the Ga-polar (0001) face of GaN, whereas for crystals doped with $\mathrm{Mg}$ (semi-insulating - see next section) it is always the opposite $\mathrm{N}$-polar (000-1) face. Therefore, doping with $\mathrm{Mg}$ (if sufficient to compensate free electrons) changes microscopic growth mechanisms on the $\{0001\}$ polar surfaces of $\mathrm{GaN}$.

If the doping level is too low and the resulting crystals are still $n$-type, the morphology is like for crystallization without doping. This suggests that the position of the Fermi level in the crystal influences the microscopic processes occurring on the growing surfaces. Such a suggestion is consistent with the results of $a b$ initio calculations $[16,17]$ showing that the formation energies of both native and impurity related point defects in $\mathrm{GaN}$ are very sensitive to the position of the Fermi level in the crystal. Figure 13 shows the cross-section scanning electron microscopy (SEM) scan of the $n$-type GaN platelet. Periodic structure is visible on the Ga-polar (0001) surface of the crystal.

Such features are often observed in many of solution grown crystals as a 


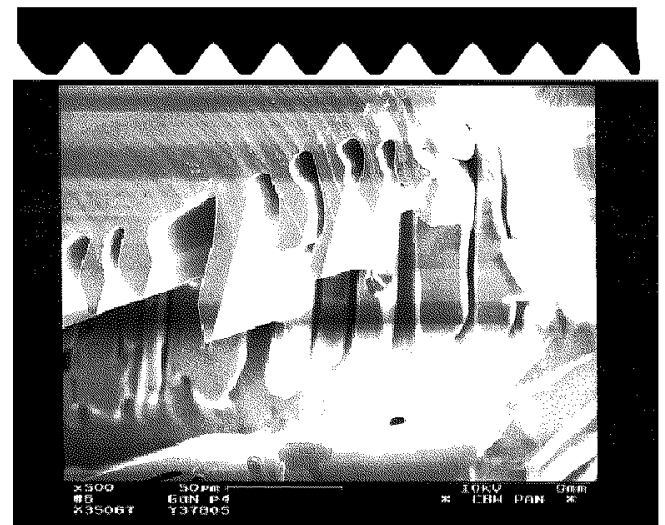

Fig. 13. Cellular growth on the Ga-polar (0001) surface of GaN crystal.

result of constitutional supercooling of the growth solution at the crystallization front. This will be explained in the next section and the method to suppress such kind of instability will be discussed.

The polarity of the crystal surfaces was identified by etching in hot alkali water solutions since the Ga-polar surface is inert to etching whereas the N-polar one etches well for both types of crystals. The method was calibrated by convergent beam electron diffraction (CBED) $[18,19]$ and X-ray photoelectron spectroscopy (XPS) [20] measurements.

From the analysis of the growth of GaN crystals without an intentional seeding it was deduced that the growth into $\langle 10-10\rangle$ directions, at the conditions corresponding to the growth rate of $0.05-0.1 \mathrm{~mm} / \mathrm{h}$, is stable and that at the same conditions, the growth into directions $\langle 11-20\rangle$ is also stable and even faster. Therefore, the size of the stable platelets should be a function of the growth time (and volume of the solution) and further scaling of the crystal size with time and the volume of the crucible is possible. It is also clear that the seeded growth into directions perpendicular to the $c$-axis should occur fast and in a stable way, especially into directions $\langle 11-20\rangle$.

Seeded growth into directions parallel to the $c$-axis seems to be much more challenging since the observed growth rates are small and the growth shows strong tendencies to be unstable. In the next section, the results of seeding are presented.

\subsubsection{Seeded growth of GaN by HNPS method}

The thin GaN platelets described in the previous section can be used for further crystallization as seed crystals. Depending on seed preparation and on the configuration of the experiment it is possible to enforce the growth in directions perpendicular or parallel to the $c$-axis of GaN. As it was already mentioned, the growth on the $\{0001\}$ polar surfaces of $\mathrm{GaN}$ is very slow and often unstable. Main features of the surface morphology like macrosteps at the edges and the periodic cellular structures suggest possible mechanisms of the unstable growth. 
For macrosteps at the edges the quite obvious reason is the accelerated growth at the edges exposed to the nitrogen flux coming from the hot part of the solution. This can be avoided by the elimination of the edges by the proper configuration of the intentional seeding experiment like it is shown in Fig. 14c. Such a configuration allows also to achieve much more uniform supersaturation across the growing surface which is very important condition for stable solution growth especially if the growing surfaces are relatively large.

The cellular growth is often a result of constitutional supercooling (Tiller et al. 1953) [21] of the solution which is graphically explained in Fig. 14a and b. If the crystallization takes place at the given crystal surface, from the solution of the concentration $C_{\mathrm{N}}$, a depleted zone at the crystallization front creates due to the incorporation of the solute into the crystal (Fig. 14a). The concentration profile at the growth front can be transformed onto the temperature profile ( $T_{\mathrm{L}}$ in the Fig. 14b) via liquidus relation. This temperature profile can be related to the real temperature gradient at the crystallization front. If the gradient is relatively small - like the one labeled $G_{\mathrm{NS}}$, the supersaturation in front of the growing surface can be bigger than at the surface itself. Then, each local acceleration of the growth results in its further enhancement, local depletion at the sides of the growing cell and the formation of a periodic cellular structure like the one shown in Fig. 13. The condition for stable growth can be expressed by the relation [22]:

$$
\frac{G_{\mathrm{L}}}{V}>-\frac{C(1-k)(-m)}{k D}
$$

where $G_{\mathrm{L}}$ is the temperature gradient at the crystallization front, $V$ is the growth rate, $k$ - distribution coefficient, $m$ - diffusion coefficient of the solute in the growth solution. The parameters at the left hand side of the relation (3) can be controlled experimentally if the configuration of the experiment corresponds to the scheme of Fig. 14a and b or c.
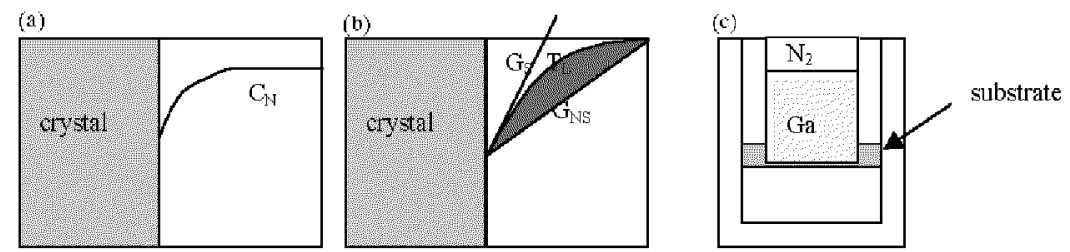

Fig. 14. Schematic illustration of constitutional supercooling at the crystallization front: (a) creation of the depleted zone at the crystallization front due to incorporation of the solute into the growing crystal, (b) different temperature gradients applied at the crystallization front related to the equilibrium liquidus temperature profile corresponding to the concentration profile of Fig. 14a, (c) configuration used for seeded growth of GaN on $\{0001\}$ polar surfaces of GaN substrates. 
The n-type GaN platelets have been used for seeded crystallization in order to suppress the cellular growth on the Ga-polar (0001) surface. The N-polar $(000-1)$ surfaces have been also used for comparison. The surfaces were prepared like for epitaxy: they have been polished mechanically and then the subsurface damage has been removed by mechano-chemical polishing for the N-polar surface [23] and by the reactive ion etching (RIE) for the Ga-polar surface.

The experiments were performed in vertical configuration similar to that from Fig. 14c. The large positive temperature gradients of the order of $100^{\circ} \mathrm{C} / \mathrm{cm}$ have been applied at the average crystallization temperature of about $1500^{\circ} \mathrm{C}$. After $20-50 \mathrm{~h}$ processes the substrates with the new crystals deposited on the $\{0001\}$ surfaces were removed from the solution and investigated. The result of the $50 \mathrm{~h}$ growth on the Ga-polar surface is shown in Fig. 15 where both the optical and SEM images of the substrate with the new grown crystal are presented.
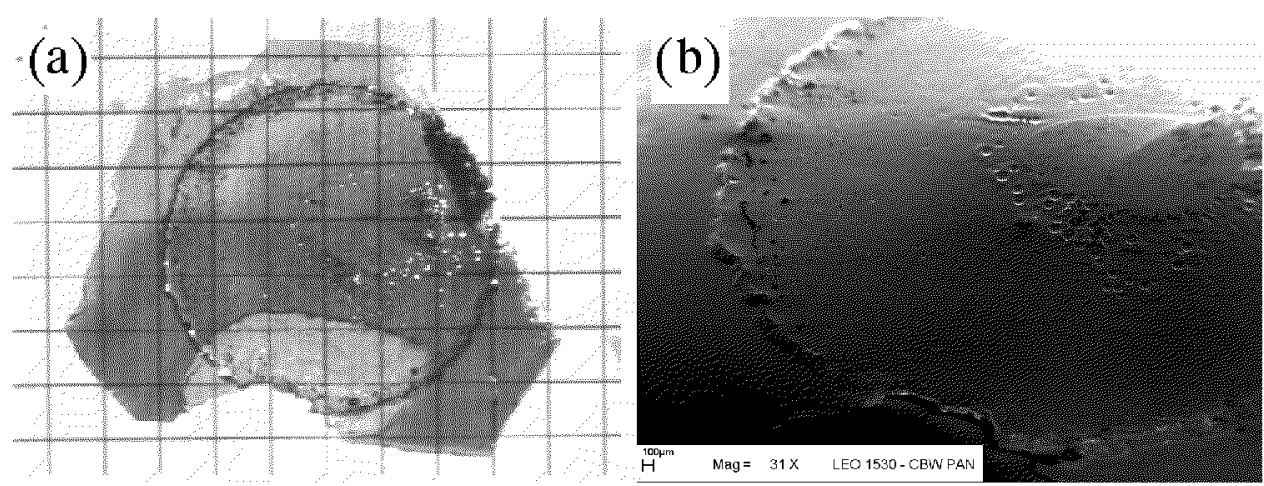

Fig. 15. GaN substrate with the new grown material on the Ga-polar surface: (a) optical image (distance between the grid lines - $1 \mathrm{~mm}$ ), (b) SEM image.

The new material was transparent, colorless, and grown as a single hillock $6 \mathrm{~mm}$ in diameter. The periodic, cellular structures were no longer present. The dominant growth mechanism was the propagation of the macrosteps from the hillock center. The position of the hillock center most probably corresponds to the minimum supersaturation being the result of the radial temperature gradients in the growth solution. The macrosteps are shown clearly in Fig. 16a and b. Such growth features are often observed for crystallization from solutions since the surface diffusion is strongly limited in comparison to the growth from the vapor phase where the growth proceeds by the propagation of monoatomic steps.

The mechanism of the growth on the N-polar surface was also the propagation of the macrosteps, however, the presence of several growth centers have been observed for the similar conditions of the experiment. It is still not clear if this is related to differences in nucleation mechanisms on the surface of different polarity or to the imperfect preparation of the surface which, in contrast to the Ga-polar one, is chemically active. 

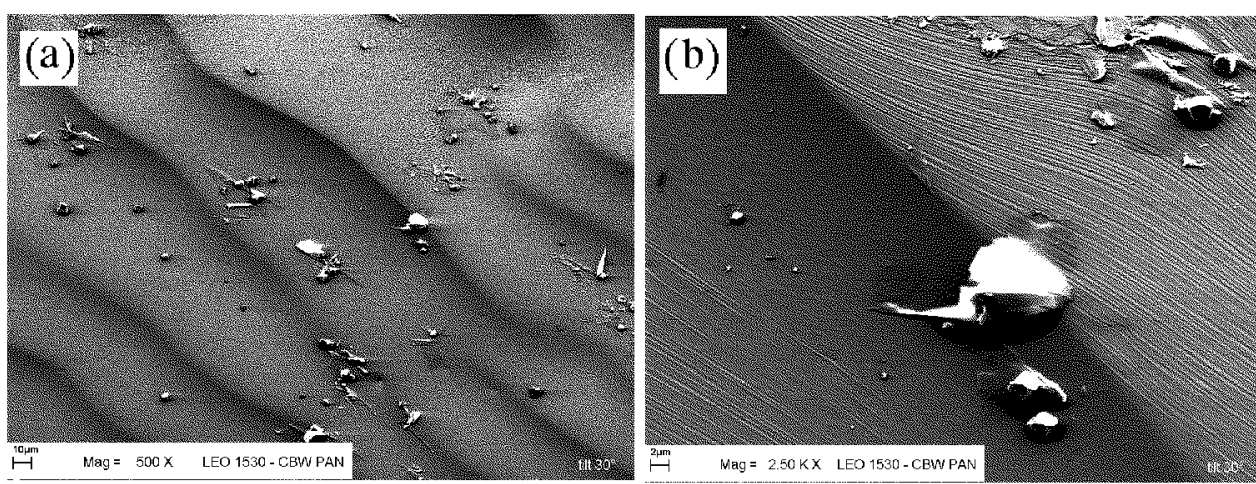

Fig. 16. Morphology of GaN crystal grown on Ga-polar surface of GaN substrate in $50 \mathrm{~h}$ process. (a), (b) SEM images of the growth hillock surface in different magnifications.

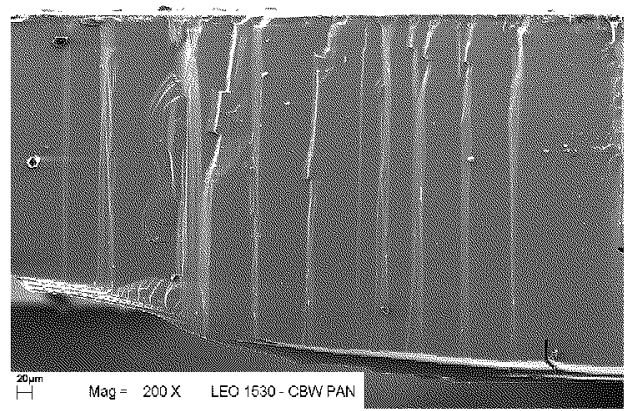

Fig. 17. SEM scan of the cross-section of GaN crystal grown on Ga surface of GaN substrate during $40 \mathrm{~h}$.

The normal growth rates observed in these experiments were $4-8 \mu \mathrm{m} / \mathrm{h}$ for both polarities, depending on supersaturation at the growth front being a function of temperature gradient and the height of Ga over the substrate. The microscopic observation of the cross-sections of the samples (Fig. 17) showed that the growth was stable in terms of continuity of the new grown material. The inclusions of the solvent and/or voids were not observed. The interfaces between substrates (80-100 $\mu \mathrm{m}$ thick) and the new grown crystals were not visible indicating that the surface preparation and the wetting procedures before seeding were performed correctly. The X-ray diffraction (XRD) analysis shows that the material grown by the method just described is of similar structural quality as the substrate used. Therefore, this kind of directional crystallization experiments will be continued to find the optimum configuration for stable growth of bulk GaN in $\langle 0001\rangle$ directions.

In particular, in order to limit the step bunching, the supersaturation across the growing surface should be more uniform. This can be achieved by the reduction of radial temperature gradients and/or by a decrease in the height of Ga over the substrate.

This method gives possibility to grow thick crystals which can be sliced into 

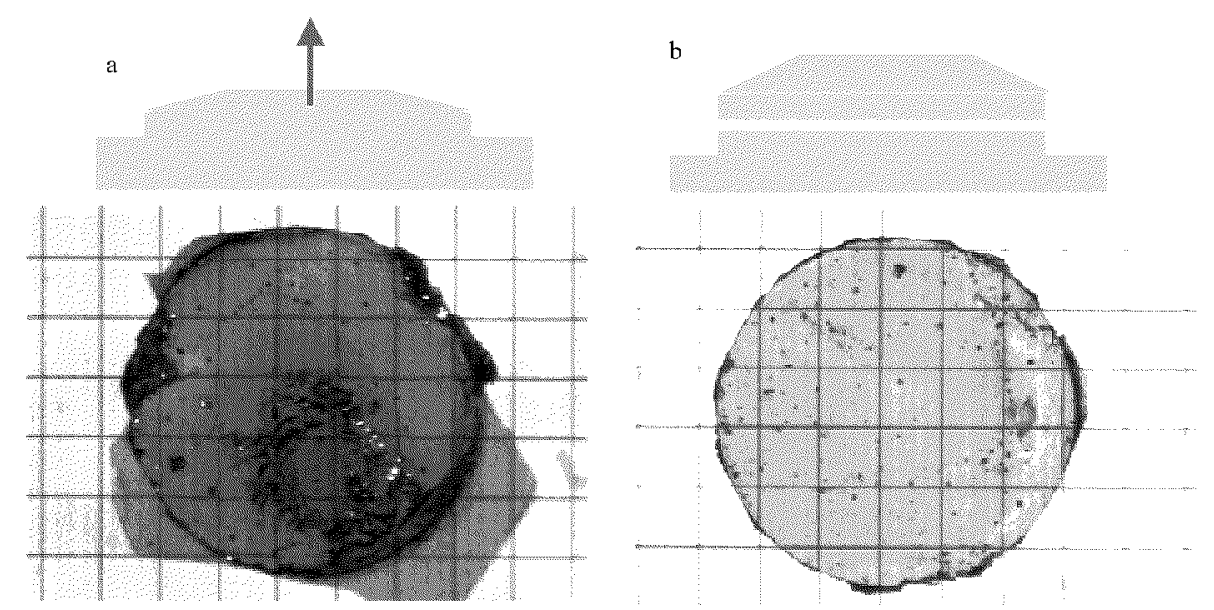

Fig. 18. GaN substrate crystal, obtained by slicing of the new material, grown by directional crystallization along c-axis. (a) Bulk GaN grown on hexagonal GaN substrate, (b) $200 \mu \mathrm{m}$ thick GaN slice.

platelets of normalized shape as shown in Fig. 18.

\subsection{Physical properties of GaN grown by HNPS method}

\subsubsection{Point defects}

As it was already mentioned, the $\mathrm{N}_{2}$ molecules dissociate at the contact with the Ga surface. However, to approach the surface they have to overcome quite high (about $3.5 \mathrm{eV}$ ) potential barrier which lowers substantially the rate of nitrogen dissociation and its further dissolution in the metal. For oxygen interacting with $\mathrm{Ga}$, there is no potential barrier for dissociation [24] and therefore even traces of this impurity in the growth system are source of the unintentional oxygen doping of GaN. Consequently, the crystals are strongly $n$-type with free electron concentration of about $5 \times 10^{19} \mathrm{~cm}^{-3}$ (metallic conductivity) and mobility of about $60 \mathrm{~cm}^{2} /(\mathrm{Vs})$ [25]. These free carriers can be fully eliminated by $\mathrm{Mg}$ acceptor added into the growth solution. Then the resistivity of the crystals becomes as high as $10^{4}-10^{6} \Omega \mathrm{cm}$ at $300 \mathrm{~K}$. Usually, the GaN:Mg crystals become p-type with an activation energy of $150 \mathrm{meV}$, at temperatures slightly exceeding $300 \mathrm{~K}$. More detailed analysis of the electrical properties of pressure grown $\mathrm{Mg}$ doped GaN crystals can be found in Ref. [26].

The presence of the native point defects in the crystals has been checked by positron annihilation measurements [27]. High concentration of $\mathrm{Ga}$ vacancies $\mathrm{V}_{\mathrm{Ga}}$ has been found in the conductive crystals in contrast to the $\mathrm{Mg}$-doped samples where no Ga-vacancies has been observed. This agreed with theoretical prediction that the formation energy of $V_{G a}$ decreases with the increase in the Fermi level energy $[16,17]$ suggesting that in the creation of these defects, thermodynamics plays a role. The difference in the photoluminescence (PL) spectra [28] of the 
conductive (strong yellow emission) and Mg-doped crystals (no yellow emission, blue $\mathrm{Mg}$-related signal) supported that $\mathrm{V}_{\mathrm{Ga}}$ is involved in yellow luminescence in GaN.

Quite different picture is observed for doping with beryllium [29]. The GaN:Be crystals are also highly resistive with the constant activation energy of $1.46 \mathrm{eV}$ at temperatures up to at least $1000 \mathrm{~K}$. But their PL spectra are dominated by a very strong yellow luminescence and the crystals contain a lot of gallium vacancies [29] like the highly conductive crystals grown without an intentional doping. Therefore, it is very probable that these crystals are $n$-type during high temperature growth and become semi-insulating only during cooling down. Such behavior can be related to the two possible configurations of Be atoms in GaN lattice ( $\mathrm{Be}_{\mathrm{Ga}}$ acceptor and $\mathrm{Be}_{\mathrm{i}}$ - donor) and their redistribution in function of temperature.

Some faces corresponding to the polar low index $\{0001\}$ and $\{10-11\}$ crystallographic planes in wurtzite structure, appearing in the pressure grown GaN crystals are non-equivalent regarding their atomic structure. This reflects in the asymmetry of the physical properties of the plate-like crystals grown without an intentional seeding.
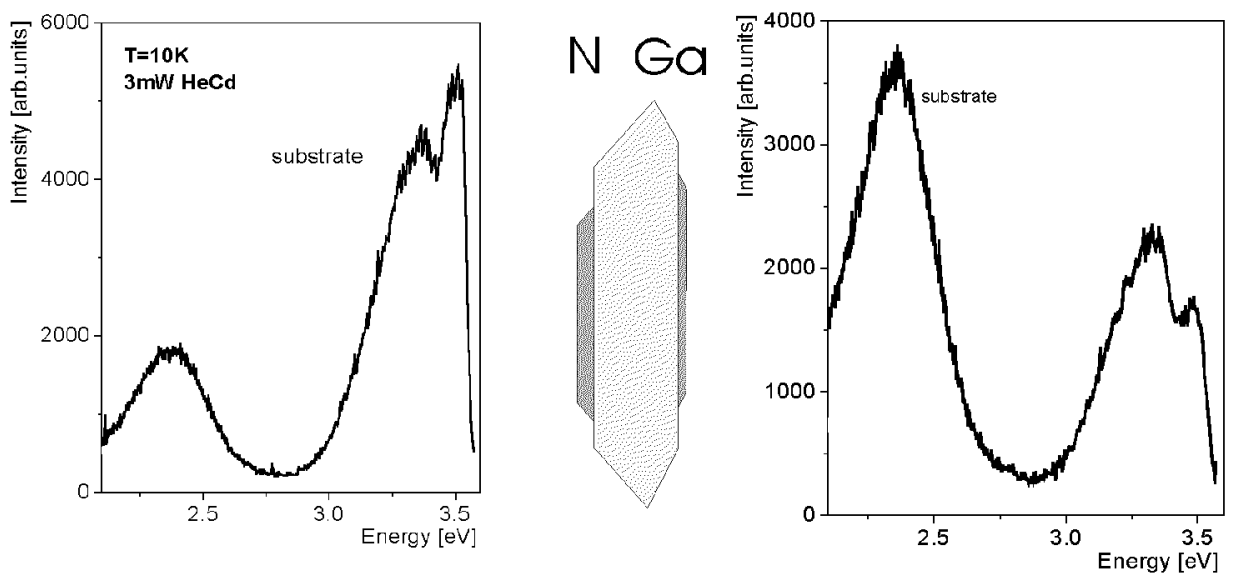

Fig. 19. Photoluminescence of GaN grown on different sides of polished platelet grown without an intentional seeding (substrate) and the platelet (substrate) itself.

In Fig. 19 there is an example of such an asymmetry. The figure shows that the material grown with $\mathrm{N}$-polarity differs in their PL properties from the material grown with the Ga-polarity which indicates that the point defects incorporate into the crystal in different ways. Additionally, the spectra coming from the material grown on the $\{0001\} \mathrm{N}$ - or Ga-polar surfaces (seeded growth) are of similar character as the spectra from corresponding surfaces of the substrate. Since the platelets were polished before being used as seeds, the suggestion arises that the platelets themselves also demonstrate a polar character despite they were grown mainly in the fastest growth $\langle 10-10\rangle$ directions which follows from their morphology. 
The strong support for that are the measurements of the free electron concentration distribution across the cleaved, as grown GaN platelet by the micro-Raman scattering technique [30]. Figure 20 shows that the border line between materials of higher (N-side) and lower (Ga-side) electron concentration is situated inside the crystal dividing it into two parts. This suggests that the microscopic (in the atomic scale) processes responsible for the formation of both native and impurity related point defects occur mainly on the $\{10-11\}$ polar faces of growing GaN crystals independently if the growth is perpendicular or parallel to the c-axis.

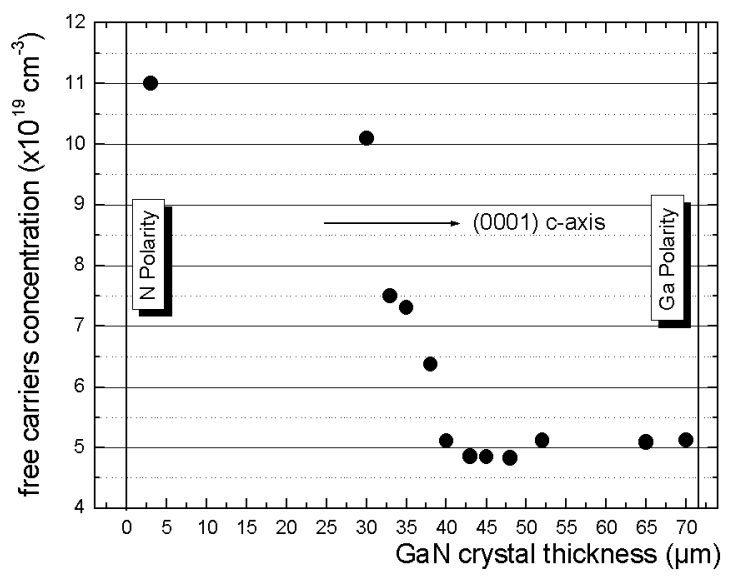

Fig. 20. Distribution of free electron concentration across the GaN platelet measured by micro-Raman scattering technique [30].

Such approach allows better understanding of the another result of positron annihilation measurements [31] showing that the concentration of Ga vacancies is much higher at the Ga-side than at the N-side of the $n$-type GaN platelets. It seems surprising at the first sight, since on the Ga-polar (0001) surface, the surface Ga atoms are bonded to the surface by three bonds whereas to the opposite one only by a one bond. If we assume, however, that the incorporation of the $\mathrm{Ga}$ atoms occurs on the $\{10-11\}$ polar surfaces, the positron annihilation results become more consistent since on the (10-11) face adjacent to the (0001) one, the Ga atoms can be bonded by one or two bonds whereas on the opposite (-101-1) polar surface adjacent to the (000-1) one, the Ga surface atoms are bonded by two or three bonds. The arrangement of the atoms on the relevant polar surfaces of GaN is shown in Fig. 21.

It follows from above that the lateral (perpendicular to the $c$-axis) growth of the platelet does not occur on the non-polar $\{10-10\}$ surfaces (at least it is not predominant) since then a plateau in the diagram of Fig. 17 should be observed. 


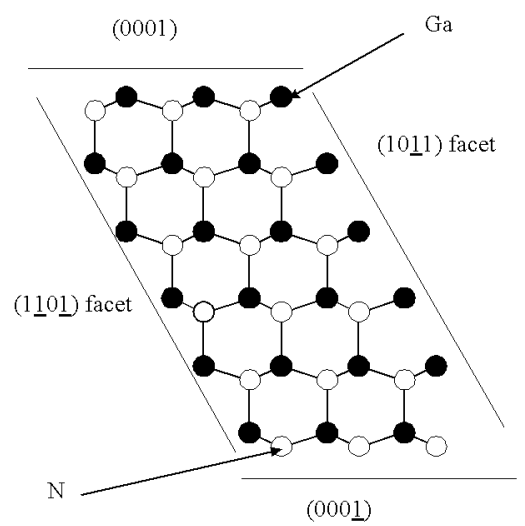

Fig. 21. The arrangement of atoms on the polar surfaces of GaN.

\subsubsection{Extended defects}

The structure of the pressure grown GaN crystals has been studied by XRD [32], transmission electron microscopy (TEM) (i.e. [18, 33-35]), defect selective etching (DSE) [35] and atomic force microscopy (AFM) of the homoepitaxial layers $[36,37]$.

In case of the conductive crystals, the shape of the X-ray rocking curves $\left((0002) \mathrm{Cu} K_{\alpha}\right.$ reflection) depends on the size of the crystal. The full widths at half maximum (FWHM) are 20-30 arcsec for $1 \mathrm{~mm}$ crystals and 30-40 arcsec for 1-3 mm ones. For larger platelets the rocking curves often split into a few $\approx 30-40$ arcsec peaks showing a presence of low angle (1-3 arcmin) boundaries separating grains of a few $\mathrm{mm}$ in size. Misorientation between grains increases monotonically from end to end of the crystal [32]. It has been suggested that this can be also related to the polar character of the platelets growth leading to some strain and its subsequent relaxation through the formation of the low angle boundaries.

It was shown by Liliental-Weber [18], by TEM examination that the N-polar (000-1) surface of the $n$-type pressure grown GaN crystals (especially for the smaller ones) is often atomically flat (2-3 monolayer steps present) and that the crystals under this surface are practically free of extended defects. Below the opposite, rough surface, a number of extended defects like stacking faults, dislocation loops, and Ga microprecipitates were observed. The relative thickness of this part usually consists of $10 \%$ of the entire thickness of the platelet. It seems that the presence of these defects is related to the growth instabilities often observed on the Ga-polar surface of the crystals grown without an intentional doping.

The detailed TEM studies of Mg-doped crystals are reported in [34]. In particular, it is shown that the introduction of $\mathrm{Mg}$ induces the formation of set of specific extended defects being mainly the stacking faults situated at the $\{0001\}$ polar surfaces of the investigated crystals. 
For the use of the crystals as substrates for epitaxy, the near surface part of the material being often the result of the unstable growth has to be removed by polishing and subsequent reactive ion etching (Ga-side) or mechano-chemical polishing (N-side) procedures. As a rule, the extended defects are not observed by TEM in the crystals used as substrates for both N- and Ga-polarity epitaxial growth. Therefore, if the epitaxy is properly performed, both the substrate and the layer quantum structure do not contain dislocations.
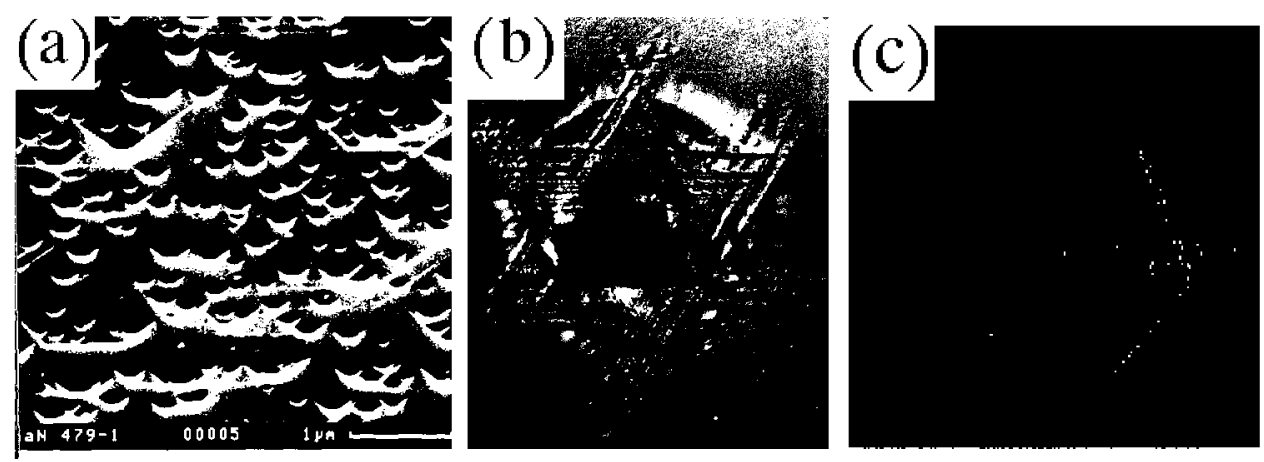

Fig. 22. Defect selective etching of GaN: (a) GaN/sapphire heteroepitaxial layer after etching in molten $\mathrm{KOH}-\mathrm{NaOH}$ eutectics [35], (b) GaN pressure grown single crystal after indentation with diamond and etching in molten $\mathrm{KOH}-\mathrm{NaOH}$ eutectics [35], the average size of the star-like pattern is $100 \mu \mathrm{m}$, (c) etch pit (EP) on the GaN crystal surface after etching in molten $\mathrm{KOH}-\mathrm{NaOH}$ eutectics [35], observed density of EP $10-10^{2} \mathrm{~cm}^{2}$.

Unfortunately, the TEM technique allows to analyze only a very small area samples. Therefore, in order to measure dislocation densities in GaN, the defect selective etching methods have been developed [35]. It was shown that etching in molten $\mathrm{KOH}-\mathrm{NaOH}$ eutectics reveals dislocations in both $\mathrm{GaN}$ heteroepitaxial layers and GaN pressure grown single crystals. The examples are presented in Fig. 22. Figure 22a shows the result of defect selective etching of typical GaN heteroepitaxial layer grown by MOCVD on sapphire substrate. High density of the etch pits is clearly visible. The same method applied for bulk crystals gives very small $\left(10-100 \mathrm{~cm}^{-2}\right)$ number of the etch pits. The typical pit observed on both heteroepitaxial layers and crystals is shown in Fig. 22c. The pattern in Fig. 22b is the result of DSE of GaN single crystal with dislocations generated intentionally by indentation with diamond. The etch pits surround the imprint of the diamond showing the area where dislocations are present and that the remaining material is dislocation-free [38]. 


\section{Pressure studies of impurities and defects in GaN and AlGaN}

\subsection{Introduction}

Pressure dependence of the band gap is one of the most important parameters characterizing a semiconductor material. In spite of the fact that this parameter describes the relative pressure shift of the conduction band (CB) with respect to the valence band (VB) of a crystal, it has been often used to determine a character of the gap of a semiconductor studied. For example, in case of $\mathrm{GaAs}-\mathrm{Al}_{x} \mathrm{Ga}_{1-x} \mathrm{As}$ system the pressure coefficient, $\mathrm{d} E_{\mathrm{G}} / \mathrm{d} P$, changes from $\approx 110 \mathrm{meV} / \mathrm{GPa}$ (in GaAs and $\mathrm{Al}_{x} \mathrm{Ga}_{1-x}$ As with $\left.x<0.4\right)$ to $\mathrm{d} E_{\mathrm{G}} / \mathrm{d} P \approx-10 \mathrm{meV} / \mathrm{GPa}$ for $x>0.4$ when AlGaAs transforms to the indirect gap semiconductor. The latter value of $\mathrm{d} E_{\mathrm{G}} / \mathrm{d} P$ characterizes the relative shift of $X$ minimum of the CB in AlGaAs system with respect to the $\Gamma$ point of the VB. The value of $\mathrm{d} E_{\mathrm{G}} / \mathrm{d} P$ for direct band semiconductor is material dependent. For most of III-V semiconductors $\mathrm{d} E_{\mathrm{G}} / \mathrm{d} P$ falls in the range 100-150 meV/GPa, whereas for more ionic II-VI's its value is much lower, i.e., between 100-50 meV/GPa [39]. Concerning nitrides, $\mathrm{d} E_{\mathrm{G}} / \mathrm{d} P$ value for $\mathrm{GaN}$ is about $40 \mathrm{meV} / \mathrm{GPa}[40]$ and is similar for AlGaN [41]. Concerning $\mathrm{d} E_{\mathrm{G}} / \mathrm{d} P$ magnitude in $\operatorname{In}_{y} \mathrm{Ga}_{1-y} \mathrm{~N}$, it seems to be significantly lower than $40 \mathrm{meV} / \mathrm{GPa}$ and dependent on In content. $\mathrm{d} E_{\mathrm{G}} / \mathrm{d} P$ decreases with an increase in $y$. However, energy of the light emitted, $E_{\mathrm{E}}$, from InGaN/GaN quantum well structures is much lower than their band gap energy. It is often observed that both $E_{\mathrm{E}}$ and $\mathrm{d} E_{\mathrm{E}} / \mathrm{d} P$ depend on the quantum well width. We will discuss this problem in the separate section of this paper.

Pressure studies of impurity/defect related states and the corresponding levels are often undertaken with a purpose of determination of the defect state character. Another situation corresponds to the use of pressure induced changes of the carrier concentration (allowing $\mathrm{CB}$ or VB to populate localized states). A metal-insulator transition, tuning of free carrier screening efficiency or creation of DX-center-like states are good examples of such procedures.

Most of the above discussed effects occur as a consequence of differences in the pressure shifts of various states $[39,42]$. In case of the hydrogenic states, which under pressure follow the changes of their parent minima, neither the ionization energy nor a carrier concentration change if pressure value is not too high. On the contrary to hydrogenic states (effective mass states, EMS), a strong pressure shift of the localized levels (with respect to CB minimum) is usually observed. It is accompanied by significant modifications in the concentration of carriers. For the localized states strongly coupled to the lattice, application of pressure at high temperatures leads, as in the previous case, to changes of the carrier concentration. However, at sufficiently low temperatures, in spite of pressure induced shift of the impurity/defect level position, no changes in density of carriers occur and the persistent photoconductivity effect is observed [43]. Barriers for electron/hole capture (and emission) prevent carrier transfer between the conduction/valence band and the localized defect state. 
The above described tendencies accompanying application of pressure were used in the experiments presented in this paper to determine a nature of the electronic state of the studied defects or to verify models proposed for their description. In particular, the following subjects are discussed: (i) pressure induced shallow to deep-localized state of donor in GaN, (ii) metastable behavior of the localized state of $\mathrm{O}$ and $\mathrm{Si}$ donor in $\mathrm{Al}_{x} \mathrm{Ga}_{1-x} \mathrm{~N}(x>0.3$ and 0.5 , respectively), (iii) possible microscopic mechanisms of the radiative recombination in GaN:Mg.

\subsection{Donors in GaN and AlGaN}

Origin of $n$-type conductivity in undoped GaN semiconductor has been discussed for a long time. Nitrogen vacancy [43] or residual donors, oxygen and silicon [44], were considered as responsible for high concentration of electrons, $n_{\mathrm{e}}$. In unintentionally doped GaN, residual oxygen is the dominant donor impurity. For $n_{\mathrm{e}}<10^{18} \mathrm{~cm}^{-3}$ (Mott transition) the Coulombic potential of the effective mass state of donors (situated $\approx 30 \mathrm{meV}$ below the $\mathrm{CB}$ ) can bind electrons if the temperature is sufficiently low. In bulk GaN crystals grown under high pressure of nitrogen from the melt of Ga the typical electron concentration is $5 \times 10^{19} \mathrm{~cm}^{-3}$ and is caused by the oxygen donor [45]. Such a high electron concentration results in metallic conductivity since all shallow O-donor states are ionized in the entire temperature range. There are several consequences of this fact. For example, (i) a coupling of the electron plasma with longitudinal optical (LO) mode leads to replacement of the LO mode by two branches of the coupled LO-plasmon modes, (ii) a strong free-carrier absorption occurs in far infrared region of the spectrum. These two effects have been used in experimental verification of the first principle calculations showing that the localized state of oxygen donor resonant with the $\mathrm{CB}$ of $\mathrm{GaN}$ can be formed [44]. Applying hydrostatic pressure causes on the one hand an upward shift of the $\mathrm{CB}$ minimum which is followed by the hydrogenic level of the shallow O-donor. On the other hand, a very weak response of the localized state to the pressure is usually observed. Thus, application of sufficiently high hydrostatic pressure introduces eventually the level caused by the localized oxygen states into the band gap of GaN and thus forces free carriers to localize on these states. They determine the ground state of the donor. As a result, the metal-insulator transition is observed at about $20 \mathrm{GPa}$. Appearance of the LO phonon mode observed experimentally at pressures higher than about $20 \mathrm{GPa}$ is illustrated in Fig. 23 [44, 45]. For the described pressure measurements diamond-anvil technique at $77 \mathrm{~K}$ was applied.

Concerning a question about more precise determination of the localized O-donor nature (and particularly a question on its coupling to the GaN crystal lattice), it has been suggested by Wetzel et al. that during the increase in the applied pressure value to the $20 \mathrm{GPa}$ two localized oxygen related states come into play. At lower pressures one-electron localizing state becomes the donor ground state, whereas at pressures above this critical range two-electron state with large 


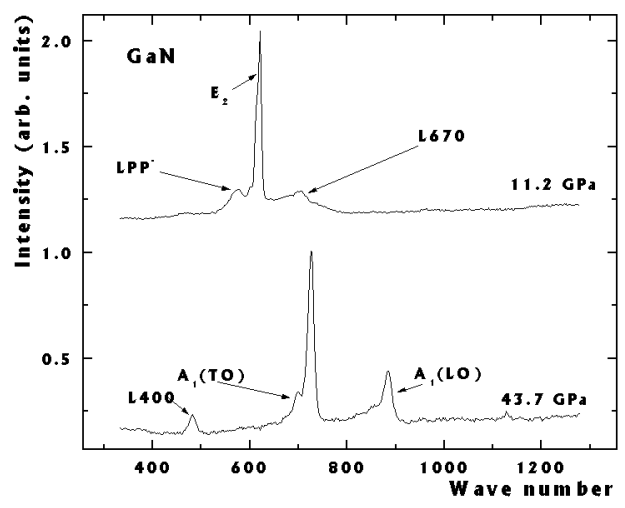

Fig. 23. Raman spectra of undoped GaN crystals.

lattice relaxation (negative- $U$ DX center) starts to localize electrons actively. It resembles the situation observed by us in GaAs doped with Ge [46].

A similar pressure "test" performed by us (up to $40 \mathrm{GPa}$ ) in GaN:Si shows an appearance of the LO mode at pressures exceeding slightly 20 GPa. It can be understood either in terms of the formation of the localized Si-donor at pressures similar to that characterizing deep O-donor or Si did not form the localized donor state in GaN. Then, the effects observed in the used GaN:Si sample is caused by the residual oxygen donors, concentration of which can be easily in the range of $10^{18} \mathrm{~cm}^{-3}$

The latter conclusion agrees with the results of calculations performed by Van de Walle [47]. Accordingly, a formation of two types of donor states has been predicted in AlGaN matrix. The first one, the effective mass state, EMS, with the level located at $\approx 30-60 \mathrm{meV}$ below the $\mathrm{CB}$ edge and the second one, localized, either resonant with $\mathrm{CB}$ for $\mathrm{GaN}$, or located in the band gap when $\mathrm{GaN}$ is alloyed with Al. This localized state of donor can have a metastable character. The metastable character of the defect is usually observed by means of the thermally activated processes of electron capture and emission onto and from the localized impurity state (thermodynamic barriers relevant at sufficiently low temperatures), as well as by the persistent photoconductivity (PPC) effect. However, it is important to note that the PPC effect can be induced not only by microscopic barriers associated with the localized impurity states. For GaN, the macroscopic barriers may originate from the modulation of conduction/valence band structure due to, for example, charged impurities and/or an inhomogeneous strain in the interface region between $\mathrm{GaN}$ and $\mathrm{Al}_{2} \mathrm{O}_{3}$ substrate [48]. The presence of such barriers causes the PPC effect which persists up to room temperature.

It was experimentally shown that for $\mathrm{Al}_{x} \mathrm{Ga}_{1-x} \mathrm{~N}$ :O with $x$ larger than about 0.3 , localized state of $\mathrm{O}$ enters the band gap [49]. For higher $x$, the material becomes highly resistive, which corresponds to the increase in the level depth with respect to the CB minimum. Moreover, it was postulated that the localized state of $\mathrm{O}$ 
exhibits metastable character in AlGaN resembling behavior of DX centers in AlGaAs [49].

Concerning character and behavior of the Si-donor related states, there are controversies in results of the theoretical predictions (see [50] and references therein). We will describe an experiment performed on $\mathrm{Al}_{x} \mathrm{Ga}_{1-x} \mathrm{~N}: \mathrm{Si}$ with $x>0.5$. In contrast to $\mathrm{Al}_{x} \mathrm{Ga}_{1-x} \mathrm{~N}$ :O which shows semi-insulating behavior for $x>0.3$, the samples doped with Si ( $x$ up to 0.5 ) are conductive. Applying hydrostatic pressure (HP) at $T=300 \mathrm{~K}$ causes a decrease in $n_{\mathrm{e}}$ which proves a localized character of the Si-related state. The energy positions obtained from fitting of temperature and pressure dependencies of $n_{\mathrm{e}}$ for the Si-related localized state vs. alloy composition are presented in Fig. 24 [50]. We included also the results from experiments of other authors performed for higher Al content $(x=0.6-1.0)$ where for Si donor the activation energy of $0.3-0.35 \mathrm{eV}$ was found $[50,51]$. Illumination of the used AlGaN:Si samples by blue light emitting diode at $T=77 \mathrm{~K}$ leads to a PPC effect which suggests the metastable character of the Si donor states populated by electrons.

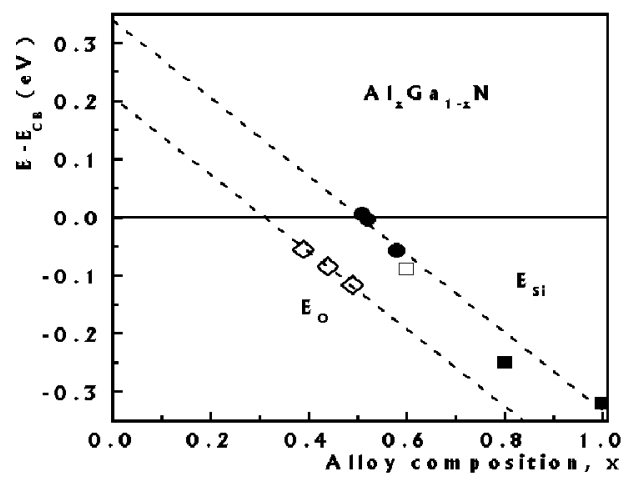

Fig. 24. Energy position with respect to the conduction band minimum of the localized $\mathrm{Si}$ and $\mathrm{O}$ related states versus alloy composition for $\mathrm{Al}_{x} \mathrm{Ga}_{1-x} \mathrm{~N}$. Dashed lines represent the data for $\mathrm{Al}_{x} \mathrm{Ga}_{1-x} \mathrm{~N}$ :Si taken from Ref. [50]. Open and full squares represent data from Refs. [52] and [51], open diamonds - data for $\mathrm{Al}_{x} \mathrm{Ga}_{1-x} \mathrm{~N}: \mathrm{O}$ from Ref. [49].

Summarizing this part of this review, we can state that the performed studies show that for $\mathrm{Al}_{x} \mathrm{Ga}_{1-x} \mathrm{~N}$ with $x>0.5$, the donor level corresponding to the Si-localized state with DX-center behavior is formed in the band gap of the alloy. This state coexists with the effective mass state. For alloys with $x$ higher than about 0.6 , the localized $\mathrm{Si}$ state becomes the ground donor state of the alloy.

\subsection{Microscopic mechanisms of the radiative recombination in $G a N: M g$}

High-pressure measurements were employed to study mechanisms of (i) $3.27 \mathrm{eV}$ emission band (and phonon replicas) and (ii) $2.9-3.1 \mathrm{eV}$ blue photoluminescence in two types of $\mathrm{GaN}: \mathrm{Mg}$ samples. GaN layers with low $\mathrm{Mg}$ doping, 
grown on bulk GaN crystals were used to study the $3.27 \mathrm{eV} \mathrm{UV} \mathrm{band.} \mathrm{This} \mathrm{lumi-}$ nescence band results from donor-acceptor pair (DAP) recombination with both impurities of shallow character. For high $\mathrm{Mg}$ doping resulting in blue light emission at about $3 \mathrm{eV}$, bulk GaN crystals were employed. For the both types of samples the pressure coefficient of the corresponding luminescence bands was measured at $77 \mathrm{~K}$ by means of diamond-anvil technique.

Figure 25a illustrates the energy shift of the zero-phonon line of DAP photoluminescence $3.27 \mathrm{eV}$ band and longitudinal phonon replicas (red shifted by about $92 \mathrm{meV}$ ) under hydrostatic pressure. It is used to determine the linear pressure coefficient characteristic of (shallow) DAP recombination (Fig. 25b). The shift is similar for all measured bands and it has a value of about $35 \mathrm{meV} / \mathrm{GPa}$, close to the pressure shift of the $\mathrm{GaN}$ band gap (about $40 \mathrm{meV} / \mathrm{GPa}$ ). It confirms the
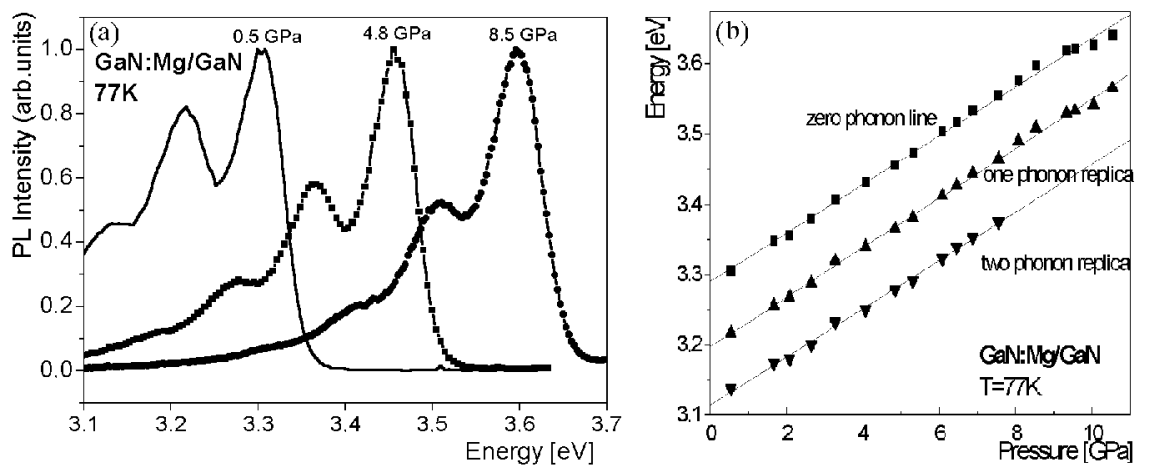

Fig. 25. DAP recombination spectra for GaN:Mg homoepitaxial layer (grown by MOVPE on bulk GaN substrate) for different pressures (a). The pressure dependence of DAP recombination in this layer (b). Solid lines represent linear fits to the experimental data corresponding to the $3.27 \mathrm{eV}$ band (zero phonon line) and its phonon replicas.

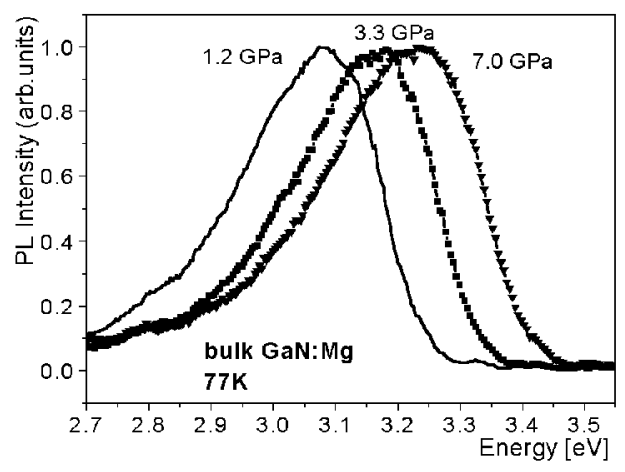

Fig. 26. Low temperature photoluminescence from $\mathrm{Mg}$ doped bulk crystal for different pressures. 
shallow character of the impurity states involved in the corresponding radiative recombination.

The 2.9-3.1 eV blue luminescence band exhibits much lower pressure coefficients of about $25 \mathrm{meV} / \mathrm{GPa}$ (Fig. 26). It shows that the initial state of the blue emission has localized character in GaN material and supplies a strong argument against models of radiative recombination in highly $\mathrm{Mg}$ doped/compensated GaN material which postulate an involvement of delocalized (shallow) electronic states as the initial ones.

\section{Epitaxy on bulk GaN}

\subsection{Introduction}

The spectacular development of GaN-based optoelectronic devices was possible due to elaboration of the two-step metal-organic chemical vapor deposition process for the growth of (GaAlIn)N epitaxial structures on highly mismatched sapphire substrates. In these structures dislocation densities are as high as $10^{8}-10^{10} \mathrm{~cm}^{-2}$ but nevertheless very efficient luminescence is possible if the structures contain InGaN [1].

It is suggested that this is due to strong localization of carriers in deep potential wells caused by compositional fluctuation in InGaN alloys, which eliminates interaction of carriers with dislocations. In this model, the influence of dislocations on the optical efficiency of InGaN can be eliminated if the distance between dislocations exceeds the size of the potential fluctuations. However, for high injection currents (i.e. in LDs), the potential minima are too shallow to contain all the injected carriers and therefore for lasers, the reduction of dislocation density in the material is much more important than for LEDs. The above model explains in a consistent way the following characteristics of Nakamura's (the unquestionable leader in the field of GaN-based technology and optoelectronics) optoelectronic devices:

- The nitride LEDs containing In in their active structures are much more efficient than LEDs with pure GaN active layers (not alloyed with In).

- InGaN LEDs' efficiency does not depend on dislocation density (if $\mathrm{Al}_{2} \mathrm{O}_{3}$ or epitaxial lateral overgrowth (ELOG) are used) in contrast to the efficiency of In-free UV diodes.

- At high injection currents, the high dislocation density lowers the efficiency even for InGaN LEDs.

Therefore, one can expect that the elimination of dislocations from the structures should lead to:

- High efficiency In-free UV LEDs.

- Higher efficiency and higher power of both UV and visible laser diodes than it is possible with dislocated structures. 
In the following both In-free and In-containing structures are considered and the results supporting the above expectations are presented.

\subsection{Metal-organic chemical vapor epitaxy on GaN substrates in HPRC Unipress}

Metalorganic chemical vapor epitaxy (MOVPE) is the most commonly used technique for growing III-N layers. The initial experience in MOVPE homoepitaxial growth on Unipress GaN substrates was gained at Warsaw University by Pakuła et al. [53], at the Wrocław Technical University by Leszczyński et al. [54], at Ulm University by Kamp et al. [55] and at CRHEA-CNRS, France by Leszczyński et al. [56]. Since 1999, when the Unipress MOVPE system was completed, almost all research on MOVPE growth on bulk GaN substrates has been done with this equipment. The MOVPE system was designed by Panek (Wrocław Technical University) and Prystawko (Unipress). It can employ either vertical or T-shaped reactors. The gases $\left(\mathrm{N}_{2}, \mathrm{H}_{2}\right.$, and $\left.\mathrm{NH}_{3}\right)$ are purified to ppb level by SAES getter purifiers. The following metalorganic compounds are used: TMG, TMA, TMI for growing (Al,Ga,In)N layers, Cp2Mg for Mg-doping, $\mathrm{SiH}_{4}$ for Si-doping. The substrate is heated by an inductive coil that induces current in a graphite (SiC-coated) susceptor. The MOVPE apparatus constructed at HPRC is presented in Fig. 27.

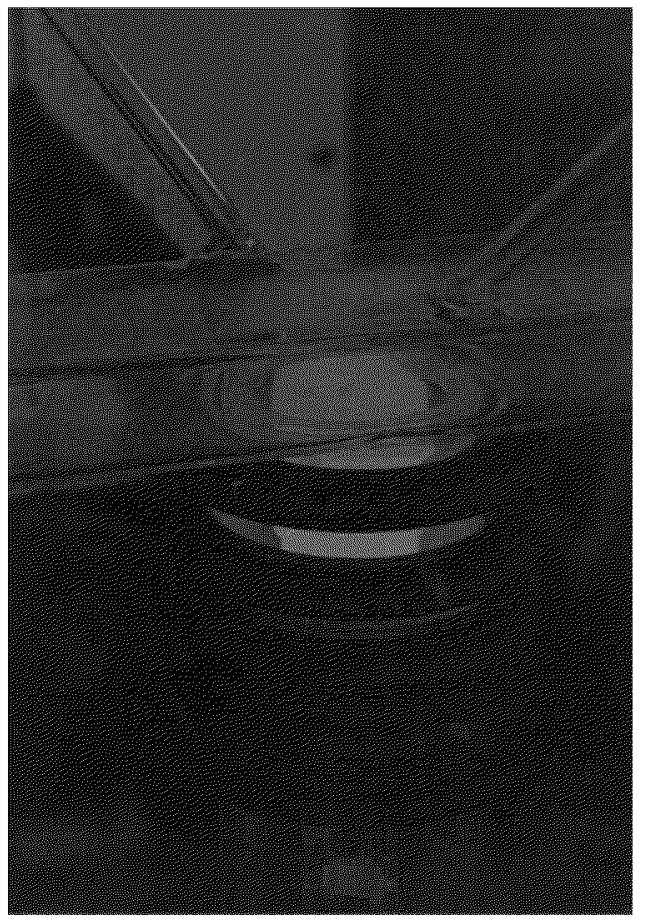

Fig. 27. MOVPE nitride apparatus in HPRC. 
The growth is monitored by laser reflectometry. A typical scan obtained during the InGaN based laser-structure growth is shown in Fig. 28. Each oscillation corresponds to about $1300 \AA$ of the growing layer. The figure shows that during a very complicated growth (more than 100 different layers) the amplitude of oscillation remains almost constant for the same chemical composition, which means that the surface does not become rough. Using laser reflectometry, it is possible to monitor the growth with $2-3 \AA$ accuracy, which is necessary for the multiple quantum wells (MQW).

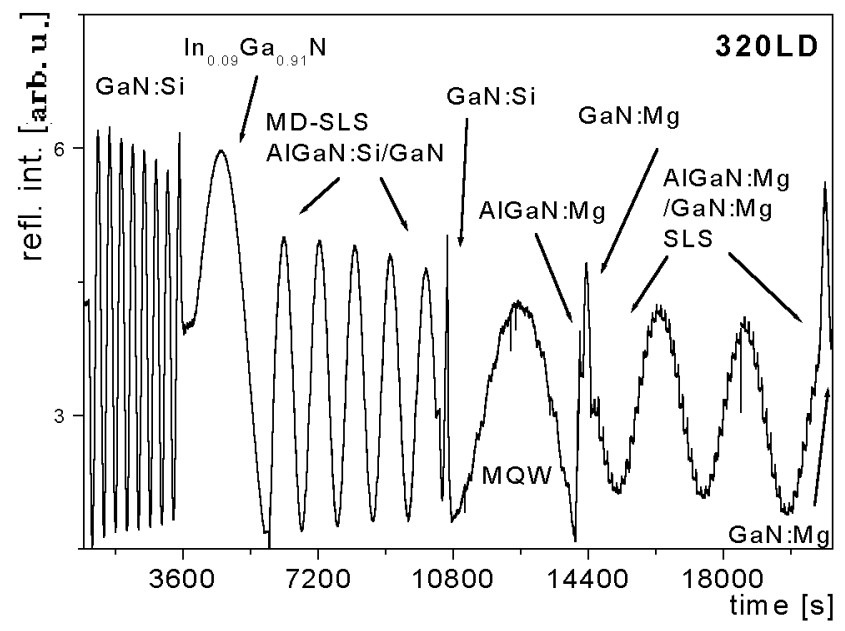

Fig. 28. Optical reflectance measured during growth of quantum structure by MOVPE.

The first important finding was that Ga-terminated (0001) face (see Fig. 10) incorporates acceptors much easier than N-terminated (000-1) face. On the contrary, N-terminated face easier incorporates donors, also impurities, like oxygen [57]. As p-doping is a crucial point for constructing optoelectronic nitride devices, Ga-face was chosen for further research. This choice created the problem of surface preparation: $\mathrm{N}$-face can be easily mechano-chemically polished, but Ga-face is chemically inert. Therefore, we had to develop the procedure of Ga-surface preparation by chemical cleaning and reactive ion etching [56].

If the surface preparation and the conditions of the epitaxial growth are right, the structure of GaN homoepitaxial layers in terms of dislocation density, follows the structure of the GaN substrates. Figure 29 compares surface morphology of GaN epitaxial layers deposited by MOVPE on GaN crystal and on GaN/sapphire substrate, in the same run.

The atomic step flow on the surface of the homoepitaxial layer is not perturbed as it is observed for GaN layer containing threading dislocations.

Therefore, the homoepitaxial GaN layers reproduce crystallographic quality of the substrates. Figure 30 shows the X-ray diffraction rocking curve that consists 

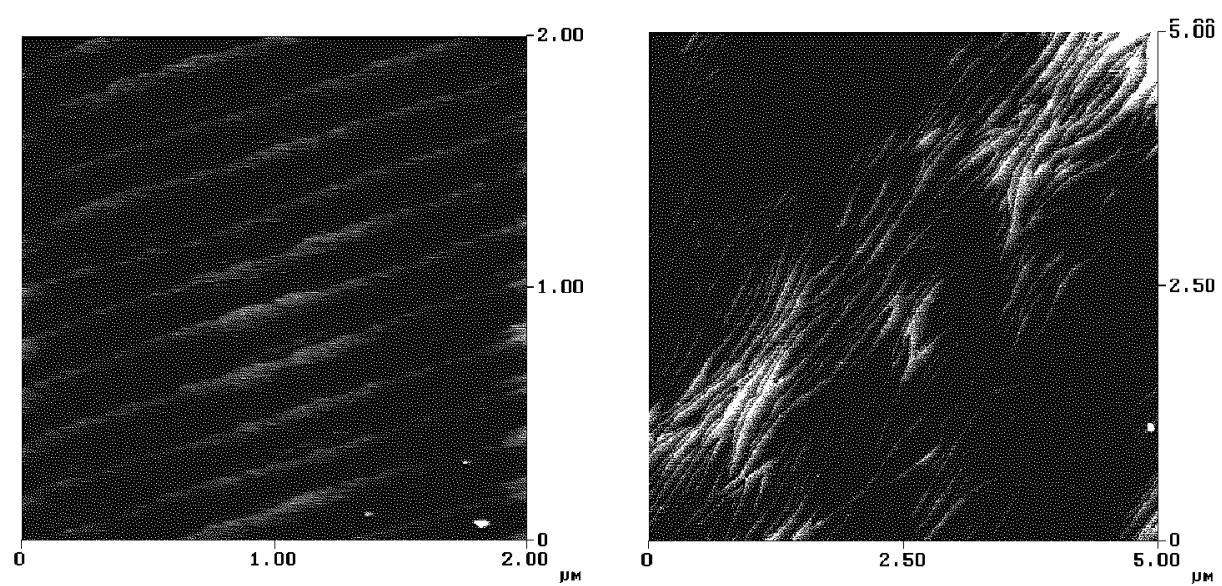

Fig. 29. Surface morphology of GaN epitaxial layers deposited by MOVPE on different substrates: (a) GaN pressure grown crystal, (b) sapphire.

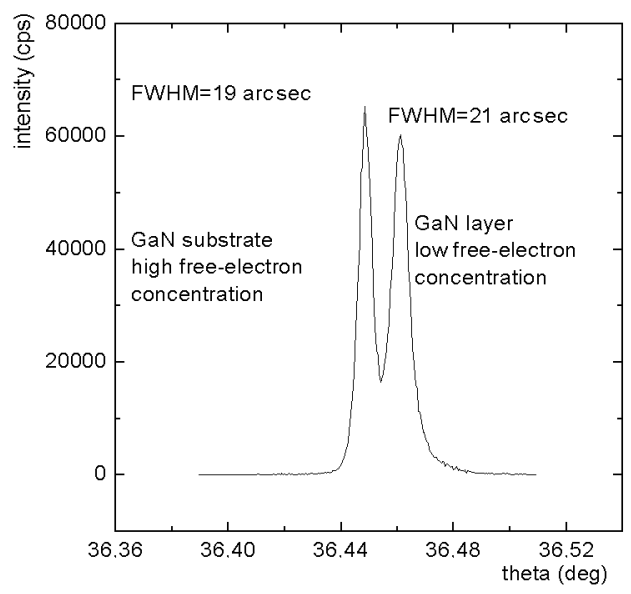

Fig. 30. X-ray diffraction rocking curve of MOVPE grown GaN homoepitaxial undoped layer.

of two peaks: one for the GaN substrate grown at high pressure, one for the homoepitaxial layer. Both peaks have FWHM (full widths at half maxima) of about 20 arcsec, which should be obtained for a perfect GaN crystal examined using this XRD system. The peaks are, however, separated because lattice of the GaN bulk crystal is slightly expanded by free electrons and point defects [58].

If a GaN homoepitaxial layer is near dislocation-free, its optical properties depend mostly on the concentration and distribution of point defects incorporated during the epitaxial growth. It is a function of the purity of the growth system, the growth conditions and the orientation of the substrate.

Due to the lack of strains and the high degree of homogeneity of the homoepitaxial GaN, the exciton related peaks in low temperature PL spectra are 
usually very narrow. The FWHM of the bound exciton lines less than $0.5 \mathrm{meV}$ is observed for layers grown by MOCVD (i.e. [59, 60]) on the Ga-polar surfaces of the GaN substrates (Fig. 31).
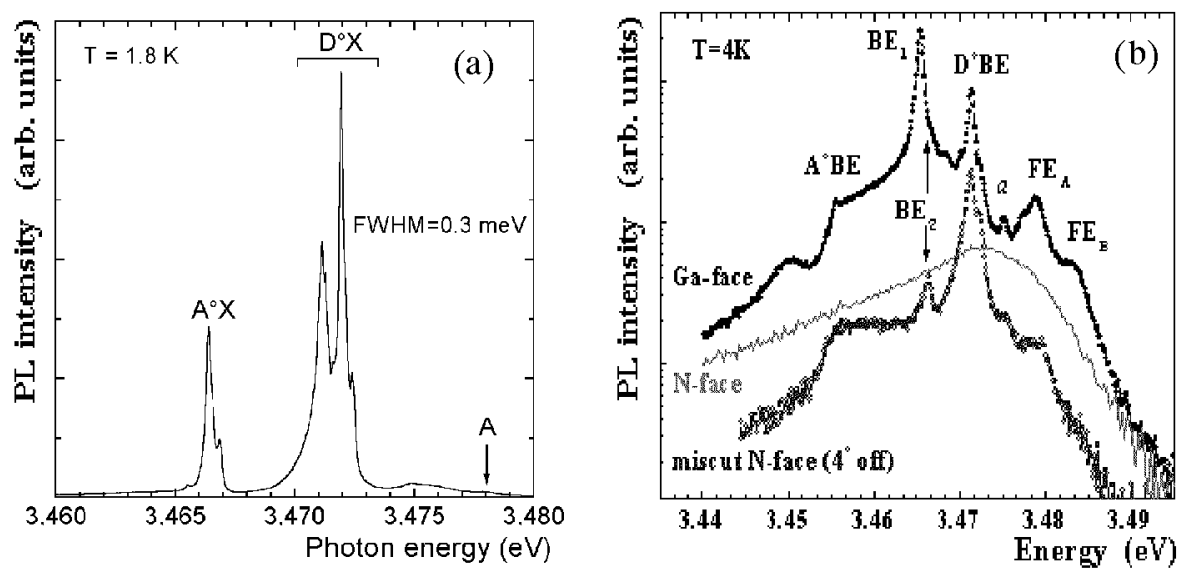

Fig. 31. Low temperature PL spectra of GaN homoepitaxial layers: (a) grown by MOVPE on the Ga-polar surface of GaN substrate, (b) grown on N-polar surfaces (exactly oriented and vicinal) of GaN substrates.

For layers deposited on the N-polar surfaces the spectra are usually much wider due to the enhanced and non-uniform incorporation of unintentional impurities on this chemically active side of GaN crystal. This can be suppressed by the use of surfaces vicinal to the $(000-1)$ as it was shown by Zauner et al. [61] who obtained narrow excitonic spectra for GaN layers grown on misoriented N-polar surfaces of the GaN substrates (Fig. 31b).

The PL decay has been also studied at high excitations [62] for GaN layers grown by MOCVD on sapphire and pressure grown GaN substrates. The decay time (at room temperature, RT) of $450 \mathrm{ps}$ measured for homoepitaxial material was 5 times longer than for corresponding heteroepitaxial layer grown at the same conditions. Figure 32 shows the decay of spontaneous luminescence measured at high excitation, close to the stimulated emission threshold for homo- and heteroepitaxial GaN.

The optically pumped low temperature (LT) stimulated emission [63] experiments confirmed that the near dislocation-free GaN is much more efficient source of light than GaN containing dislocations. The stimulated emission in GaN homoepitaxial layer grown by MOCVD started at the excitation power density lower than $1 \mathrm{MW} / \mathrm{cm}^{2}$ whereas for GaN grown on $\mathrm{SiC}$ only at $10 \mathrm{MW} / \mathrm{cm}^{2}$. The stimulated emission peak for the homoepitaxial layer was as narrow as about $5 \mathrm{meV}$ (about $40 \mathrm{meV}$ for $\mathrm{GaN} / \mathrm{SiC}$ ). The InGaN near dislocation-free epitaxial layers and quantum wells were grown on the pressure grown crystals by MOVPE by 


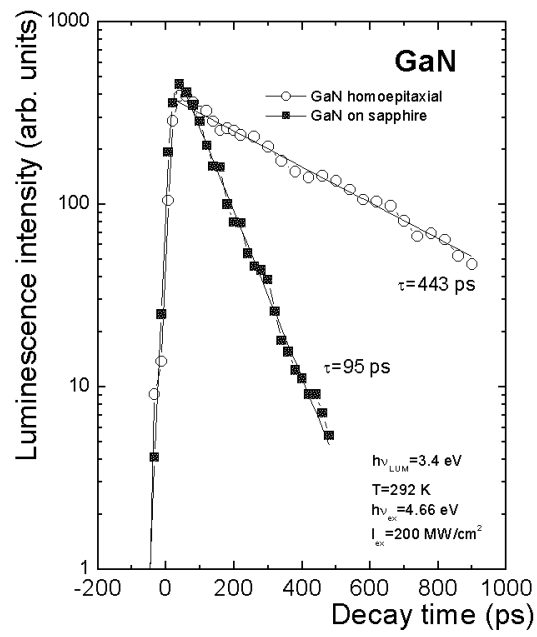

Fig. 32. Spontaneous luminescence transients for GaN films grown on sapphire (solid squares) and on GaN substrates (open circles) [62].
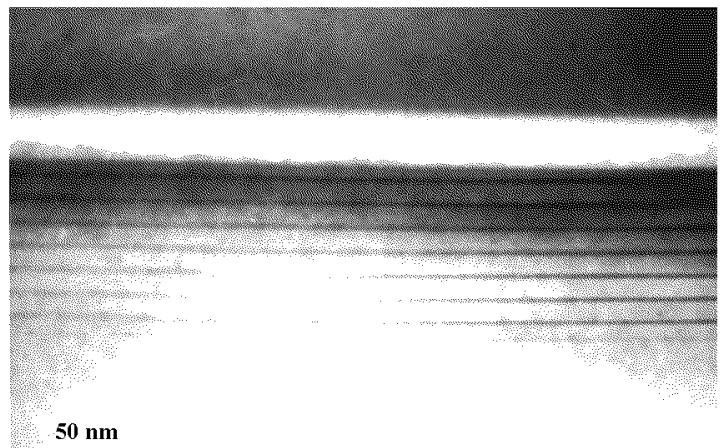

Fig. 33. InGaN MQW grown on the Ga-polar (0001) surface of GaN substrate by MOCVD [36], TEM - courtesy of M. Albrecht.

Leszczyński et al. [64], in High Pressure Research Center. In Fig. 33 the TEM images of one of the InGaN MQWs grown in Warsaw is shown to demonstrate that the structures are dislocation-free.

Dislocations in the structures grown on the almost defect-free substrates can obviously appear as a result of the lattice mismatch between $\mathrm{GaN}$ and its ternaries InGaN and AlGaN. This was analyzed by Leszczyński et al. [64] by the X-ray measurements of lattice parameters of various GaN-based epitaxial layers deposited on GaN substrates in function of InGaN (AlGaN) composition and thickness. It was shown that the boundary between fully strained and relaxed InGaN (AlGaN) layers is in a safe distance from mismatch - thickness area for layers necessary for a typical blue laser structure. These results are collected on the diagram in Fig. 34a. Figure 34b shows an example of the multilayer structure similar to the 

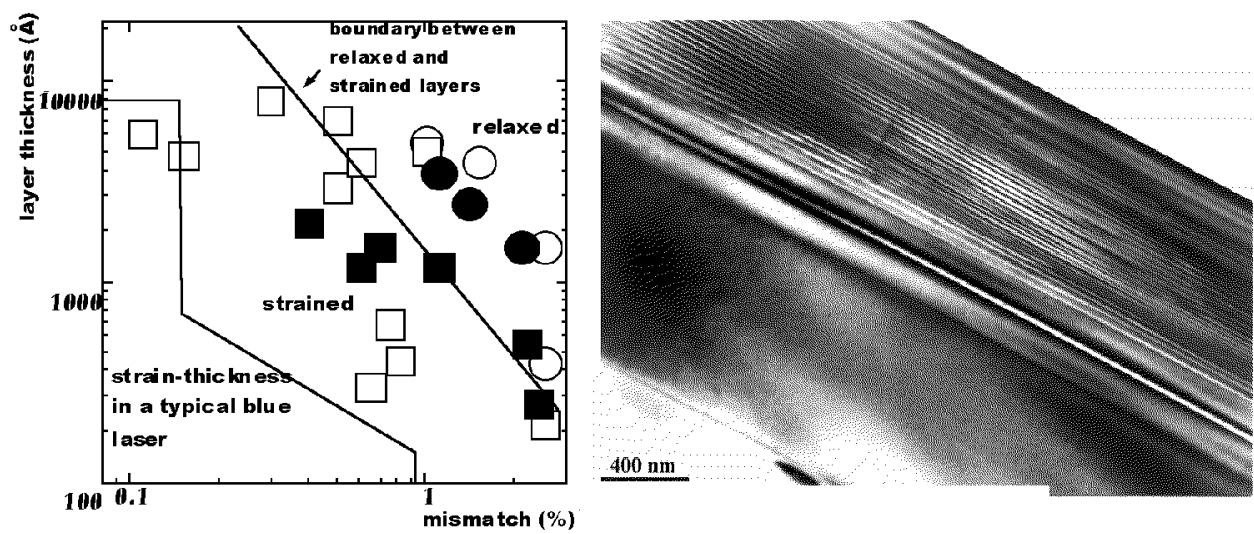

Fig. 34. Critical conditions for III-N ternaries: (a) X-ray data: open circles AlGaN relaxed, open squares - AlGaN strained, filled squares - InGaN strained, open squares - InGaN relaxed, (b) TEM image of the multilayer structure deposited on GaN substrate by MOCVD, the sequence of layers from the lower left corner: $n$-GaN, $n$ - $\mathrm{Al}_{0.11} \mathrm{Ga}_{0.89} \mathrm{~N} / n$-GaN superlattice, $n$-GaN, $\mathrm{In}_{0.09} \mathrm{Ga}_{0.91} \mathrm{~N}, p-\mathrm{GaN}$, $p$ - $\mathrm{Al}_{0.14} \mathrm{Ga}_{0.86} \mathrm{~N} / p$-GaN superlattice, $p$-GaN ((b) - courtesy of M. Albrecht).

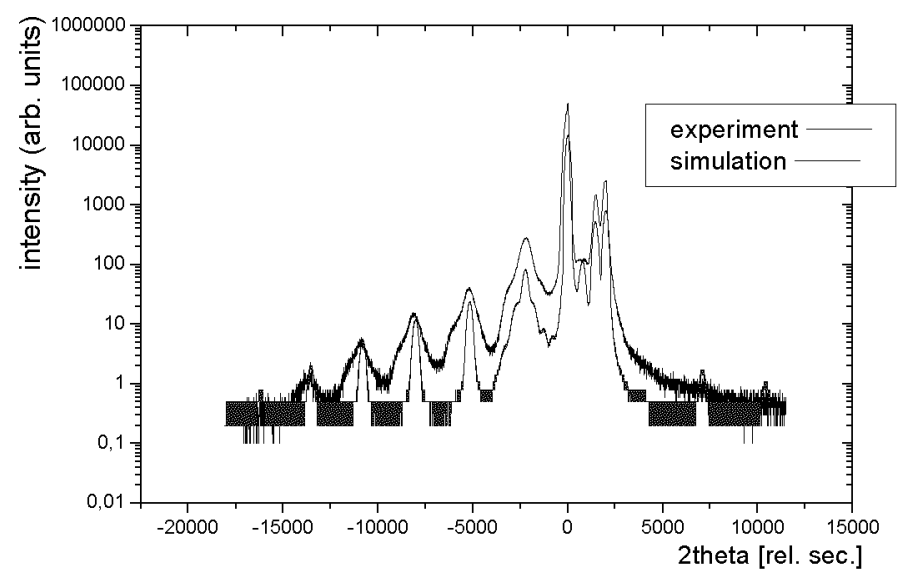

Fig. 35. X-ray scan of laser structure. For comparison an X-ray intensity of simulated perfect structure is presented.

full structure of blue laser. The example confirms that no mismatch dislocations are generated if the structure is grown on dislocation-free GaN substrate.

TEM and AFM techniques can probe only an area of a few square microns. Using XRD we examined an area of a few millimeters squared. Figure 35 presents an experimental scan for a laser structure compared to a theoretical simulation using a dynamical X-ray diffraction theory.

For GaN and AlGaN layers (on the right hand side of the scan), the peaks are very narrow, not broadened by compositional fluctuations. For InGaN, as it is 
observed by all growers, the peaks are slightly broader than for a perfect structure.

\subsection{Molecular beam epitaxy}

Nitride research by MBE started to develop substantially later than MOVPE. But due to higher purity, better interface quality, and easier $p$-doping MBE has become a widely used tool in research on nitrides. As in epitaxy on bulk GaN we do not need LT buffer layers, the MBE method seems to be especially suited for deposition of nitrides on dislocation-free substrates.

The MBE experiments conducted in collaboration with Ulm University (M. Kamp), CRHEA (J. Massies and N. Grandjean), Minnesota University (R. Helm) and Nottingham University (T. Foxon) gave many results which confirmed that combination of the dislocation-free GaN substrates and MBE can give top-performance device structures.

The MBE technique has been used mainly for the growth of GaN/AlGaN structures [65-68]. Near dislocation-free GaN/AlGaN multi-quantum wells and structures have been grown on the N-polar surfaces of GaN crystals by RF plasma assisted MBE (PA MBE) [65,66] and on the Ga-polar surfaces by MBE with $\mathrm{NH}_{3}$ nitrogen source (R MBE) $[65,66]$. As a rule, the optical properties of the structures without dislocations were much better than for similar structures grown at the same conditions, on sapphire.

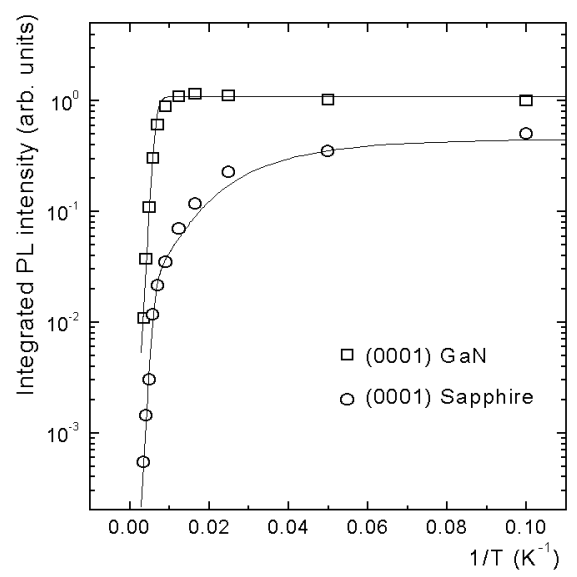

Fig. 36. Temperature dependence of the integrated PL intensity of homoepitaxial (squares) and heteroepitaxial (circles) $\mathrm{GaN} / \mathrm{Al}_{0.1} \mathrm{Ga}_{0.9} \mathrm{~N}$ QWs [69].

For example in Fig. 36, the integrated intensities of the PL coming from similar structures (8 ML GaN single QW (SQW) with the $\mathrm{Al}_{0.1} \mathrm{Ga}_{0.9} \mathrm{~N} 50 \mathrm{~nm}$ barriers) grown by R MBE [69] on sapphire and bulk pressure grown GaN substrates are compared. It is shown that for the structure deposited on GaN, the PL is much stronger, especially at RT. For the structure grown on GaN substrate, the PL intensity starts to decrease with temperature only at about $100 \mathrm{~K}$ mainly 
due to the thermal escape of carriers from the quantum well towards the AlGaN barriers, as was observed for classical III-V QW heterostructures with dislocations densities lower than $10^{3} \mathrm{~cm}^{-2}$ [68]. For heteroepitaxial structure, the presence of dislocations strongly influences the non-radiative recombination processes. The PL intensity starts to decrease at much lower temperatures due to delocalization of excitons and their further interaction with dislocations.

For devices on dislocation-free substrates, it is especially important that further increase in the emission efficiency can be achieved by increasing the $\mathrm{Al}$ content in the barriers. This opens the possibility for construction of highly efficient UV LEDs and lasers.

The semi-insulating GaN:Mg substrates have been used for growth GaN/ AlGaN heterostructures with two-dimensional electron gas (2DEG) by RF MBE [67]. The Hall mobility for $2 \mathrm{DEG}$ as high as $60100 \mathrm{~cm}^{2} /(\mathrm{V} \mathrm{s})$ at $1.5 \mathrm{~K}$ (one of the highest reported for GaN) has been measured. The Shubnikov-de Haas oscillations starting at $1.8 \mathrm{~T}$ and well-defined quantum Hall effect (QHE) indicated high quality of the MBE grown heterostructure - Fig. 37.

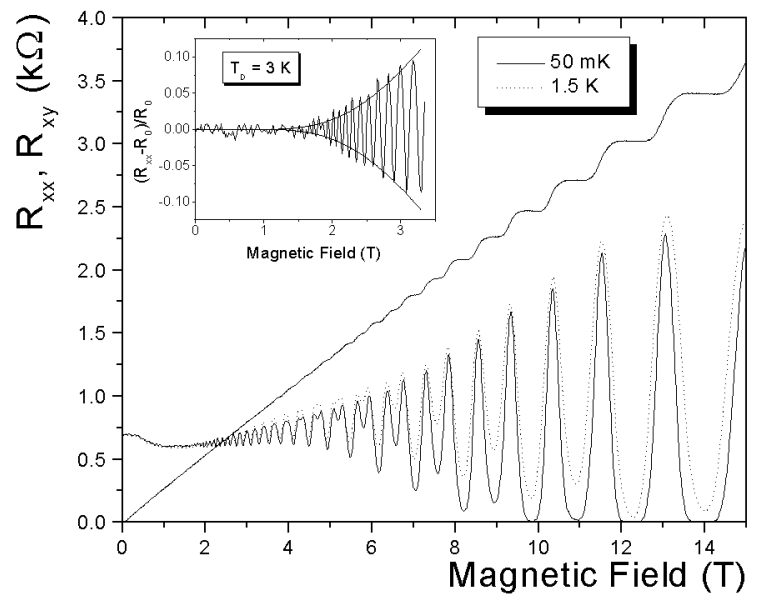

Fig. 37. Longitudinal $\left(R_{x x}\right)$ and transverse $\left(R_{x y}\right)$ magnetoresistance versus magnetic field at $1.5 \mathrm{~K}$ and $50 \mathrm{mK}$. Shubnikov-de Haas oscillations ( $\mathrm{SdHO}$ ) begin at $1.8 \mathrm{~T}$. The insert shows the low-field part of the SdHO after the normalization by the low-field resistance value, $R_{0}$.

From the analysis of the scattering processes it was suggested that in such near dislocation-free structure the ionized impurity scattering was the dominant low temperature mobility limiting mechanism.

If the substrate is dislocation-free, the strain relaxation processes in low misfit heteroepitaxy is unaffected by the presence of threading dislocations. Both elastic and plastic relaxation has been studied [65] for GaN/AlGaN multilayer structures grown by plasma assisted MBE on GaN pressure grown substrates. It 


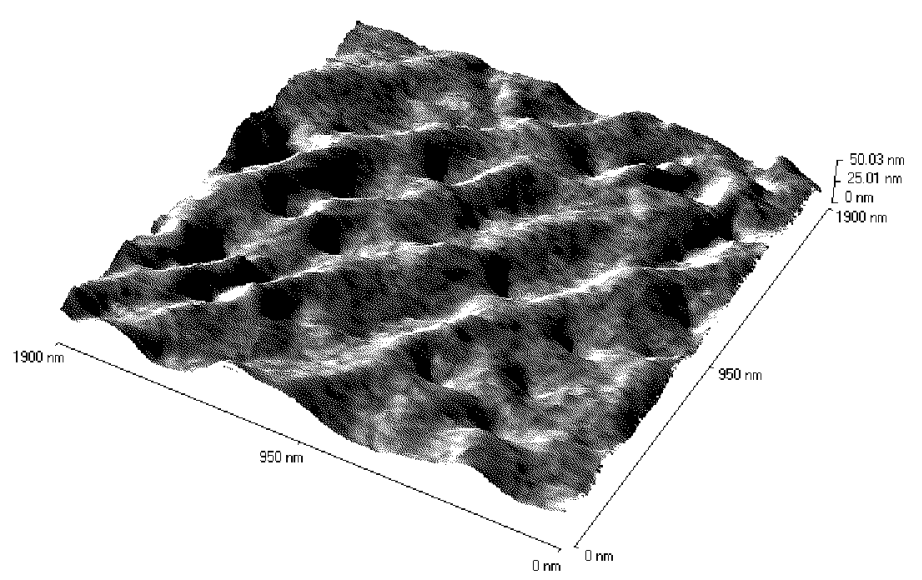

Fig. 38. Surface morphology (AFM - courtesy of R. Campion) of $40 \mathrm{~nm} \mathrm{Al}_{0.1} \mathrm{Ga}_{0.9} \mathrm{~N}$ layer grown by plasma assisted MBE on N-polar surface of GaN substrate.

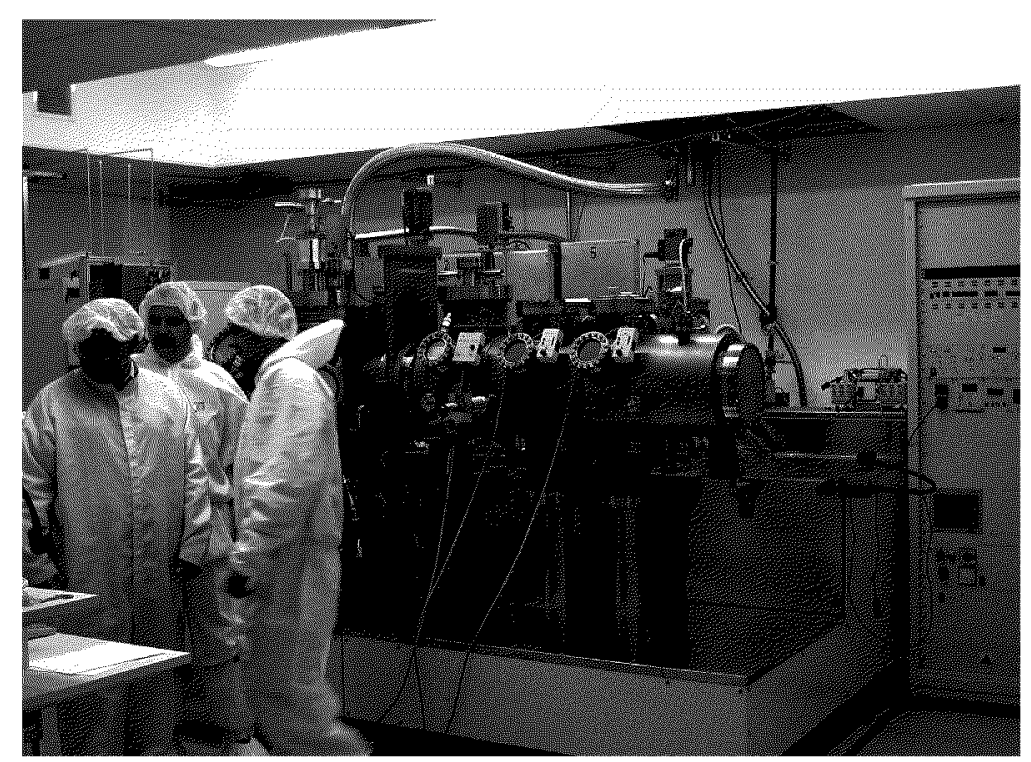

Fig. 39. MBE growth apparatus, in HPRC.

was shown that the crystal growth and relaxation in III-nitrides is not different from conventional III-V systems. The elastic strain relaxation by sinusoidal undulation of the AlGaN layers has been observed above a critical thickness depending on the Al content. An example of the AlGaN surface morphology with the sinusoidal undulation is shown in Fig. 38. The results obtained in [65] indicate new possibilities for designing low misfit heteroepitaxial growth including self-organized formation of quantum structures.

The results of the MBE deposition of nitride structures on dislocation-free 
substrates were excellent stimulation to continue the research. Figure 39 shows the MBE V-100 machine installed recently in HPRC to develop the optimum methods of growing dislocation-free nitrides especially those based on In-free GaN/AlGaN quantum structures. This machine will also allow to improve the electrical properties of $p$-type layers in quantum well structures.

\section{Electric field effects in GaN based quantum structures - pressure studies}

Contribution of the built-in electric field to the properties of wurtzite group-III nitrides has been widely disputed during last few years [70-72]. The considered materials exhibit pyroelectric properties and strong piezoelectric character which leads to the appearance of the electric polarization. It consists of two contributions: (i) piezoelectric polarization due to strain induced by a lattice-mismatch $[73,74]$ and (ii) interface charge accumulation between epitaxial layers of GaN based alloys caused by the spontaneous polarization. The latter effect is present even in the bulk nitrides and originates from a relative shift of the cation and anion sublattices along $c$-axis of the wurtzite polar structure [70]. Effects resulting from the built-in macroscopic polarization can entirely determine some basic properties of nitrides. In case of nitride systems consisting of the layers crystallizing in the cubic structure neither spontaneous nor piezoelectric polarizations are present in one of the equivalent $\langle 100\rangle$ directions.

In wurtzite nitride structures the effective electric field, directed along $c$-axis (epitaxial growth direction) caused by (i) and (ii) leads to a bending of the potential profiles related to the conduction and valence bands in quantum structures consisting of InGaN/GaN or GaN/AlGaN heterostructures. As a result, the spatial separation of excited electrons and holes takes place which is accompanied by the decrease in their energetic separation. This represents the quantum confined Stark effect (QCSE). The fingerprint of the QCSE is its dependence on the quantum well thickness. In the other words, with increasing the quantum well width the spontaneous emission energy, $E_{\mathrm{E}}$, decreases. This is illustrated in Fig. 40 where the PL of AlGaN/GaN/AlGaN QW with different GaN thickness is shown. The energy positions of PL from corresponding quantum wells are red-shifted due to the internal electric fields. For the thickest 16 monolayers (ML) GaN quantum well it is even lower than PL from bulk GaN.

The same effect is observed when the built-in electric field increases. Moreover, the other consequence of this effect is a difference in energies characterizing the light emission and absorption, i.e., Stokes shift, which is usually observed in the considered quantum structures.

The other effect leading to a red shift of the emitted light energy, as well as to the Stokes shift originates from the fluctuations in the In composition in quantum well or epitaxial layer of InGaN $[73,75]$. This effect seems to be omnipresent and 


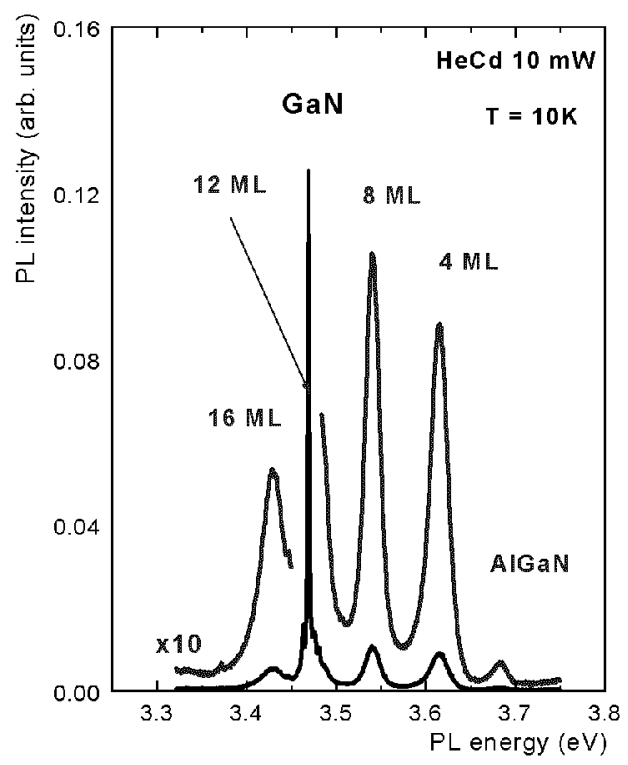

Fig. 40. Low temperature PL of AlGaN/GaN/AlGaN QW of various width. For comparison PL of bulk GaN is shown.

has been reported in hexagonal as well as cubic InGaN structures [76]. Formation of (pseudo)quantum dots corresponding to regions of higher In concentration has been invoked by many researchers. These In content fluctuations are believed to result from the effect of a phase separation governing the formation of InGaN alloy $[77,78]$, particularly for $\operatorname{In}_{x} \mathrm{Ga}_{1-x} \mathrm{~N}$ with higher $x$. In the epitaxial layer of InGaN, concentration of In cations can locally differ significantly from the average, technologically predetermined value. Similarly to the optical effects found in InGaN layers/structures subject to the internal electric fields ((i) and (ii)), in case of In content fluctuations, the light emission occurs in regions of lower energy gap which in this case corresponds to higher In content (pseudo-dots). Localized electronic states (excitons) are likely involved here.

All above mentioned effects make an unambiguous determination of band gap magnitude, $E_{\mathrm{G}}$, versus $x$ in $\operatorname{In}_{x} \mathrm{Ga}_{1-x} \mathrm{~N}$ difficult. However, it is widely accepted now that the band gap bowing parameter for this alloy is large. This reflects the strong drop of the $E_{\mathrm{G}}$ value for $\operatorname{In}_{x} \mathrm{Ga}_{1-x} \mathrm{~N}$ with low In content $(x \leq 0.25)$ [79]. An increase in the Stokes shift and a commonly reported broadening of the absorption edge in $\operatorname{In}_{x} \mathrm{Ga}_{1-x} \mathrm{~N}$ alloys accompany a rise in $x$ value.

The other puzzling observation pointing out a particular role of In in InGaN alloys, consists in the finding a small and strongly In-content dependent pressure coefficient of the light emission energy, $E_{\mathrm{E}}$, in thick layers as well as quantum structures of hexagonal $\operatorname{In}_{x} \mathrm{Ga}_{1-x} \mathrm{~N}[80,81]$. Our previous studies showed that with increasing $x, \mathrm{~d} E_{\mathrm{E}} / \mathrm{d} P$ decreases drastically. For example, for $x \approx 0.2$ the 
pressure coefficient $\mathrm{d} E_{\mathrm{E}} / \mathrm{d} P \approx 15 \mathrm{meV} / \mathrm{GPa}$ has been found in the $3.5 \mathrm{~nm}$ thick InGaN quantum well. It is almost three times smaller than that measured for $\mathrm{GaN}(40 \mathrm{meV} / \mathrm{GPa}$ ) [82] and much lower than the pressure shift of the band gap calculated for $\operatorname{InN}(\approx 25-30 \mathrm{meV} / \mathrm{GPa})[83,84]$.

In this work we describe results of two different experiments performed by us very recently with the purpose of elucidation of the microscopic mechanism responsible for low value of the light emission shift with pressure in quantum structures of group-III nitrides.

Experiment 1. The pressure studies of light emission in the series of quantum wells of hexagonal $\operatorname{In}_{x} \mathrm{Ga}_{1-x} \mathrm{~N}(x \approx 0.2)$ with different quantum well (QW) widths are reported [85]. An assumption about the involvement of the electric field (and its modification) to the pressure variation of the light emission energy leads to the strong and linear dependence of $\mathrm{d} E_{\mathrm{E}} / \mathrm{d} P$ on the width of quantum wells used for pressure studies. We mentioned an associated increase in the QCSE above.

We have studied the set of six InGaN/GaN samples consisting of a quantum well of $\operatorname{In}_{x} \mathrm{Ga}_{1-x} \mathrm{~N}(x \approx 0.2)$ embedded between GaN barriers. The samples used differ by the well thickness which changes from $1 \mathrm{~nm}$ to $5 \mathrm{~nm}$. They were grown by MBE at CRHEA Laboratory, Valbonne, France. Figure 41 illustrates the photoluminescence spectra obtained in the samples used.

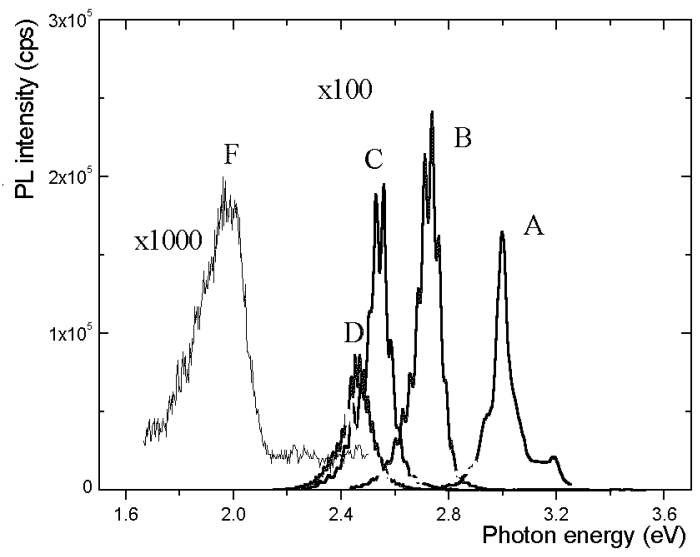

Fig. 41. Photoluminescence of $\mathrm{Ga}_{0.8} \operatorname{In}_{0.2} \mathrm{~N} / \mathrm{GaN}$ structures of different thickness: $A$ $h=1 \mathrm{~nm} ; B-h=2 \mathrm{~nm} ; C-h=2.5 \mathrm{~nm} ; D-h=3.5 \mathrm{~nm} ; E-h=4 \mathrm{~nm} ; F-$ $h=5 \mathrm{~nm}$.

Energy of the emitted light decreases linearly with the increase in the well width in the range between $3 \mathrm{eV}$ and $1.8 \mathrm{eV}$. Very similar result has been obtained in studies of the PL energy dependence on the applied hydrostatic pressure [85]. Figure 42 shows the linear decrease in the pressure shift of the photoluminescence energy with the increase in the well thickness [85]. The both effects strongly suggest 


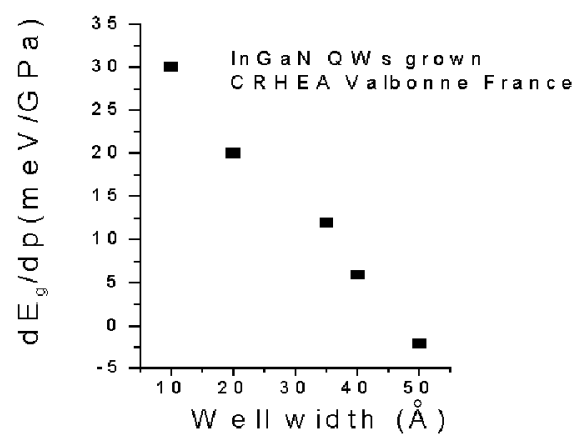

Fig. 42. The dependence of pressure shift of PL with the increase in wall thickness.

an involvement of the electric polarization in the studied samples. Strong arguments supporting this interpretation have been supplied by Vaschenko et al. [86] who reported on the pressure induced increase in the photoluminescence decay time, $\tau$, in QWs of InGaN/GaN. This effect corresponds to the spatial separation of the wave functions of recombining electron and hole pairs due to the increase in a strong electric field (QCSE) in the studied structures.

However, a standard approach to pressure induced changes of the built-in electric field in InGaN/GaN quantum structures leads to the effect opposite to the described above. This approach neglects for example the nonlinear changes of the piezoelectric tensor with strain. The necessity of taking into account such modifications of the piezoelectric tensor has been shown by Vaschenko et al. [87] and Łepkowski et al. [88]. As a result, an increase in the piezoelectric polarization with the applied pressure and consequently a decrease in $\mathrm{d} E_{\mathrm{E}} / \mathrm{d} P$ results from the performed calculations.

Experiment 2. The purpose of this study was to examine the effect of pressure on light emission, $E_{\mathrm{E}}$, from $\mathrm{GaN} / \mathrm{AlGaN} \mathrm{QWs}$ and to answer two important questions: (i) what is the value of $\mathrm{d} E_{\mathrm{E}} / \mathrm{d} P$ in the system with binary $\mathrm{QW}$ and (ii) to which extent a magnitude of $\mathrm{d} E_{\mathrm{E}} / \mathrm{d} P$ in GaN/AlGaN QWs is determined by pressure induced modifications of the built-in electric field (as seems to be a dominant factor for $\mathrm{d} E_{\mathrm{E}} / \mathrm{d} P$ in InGaN/GaN QWs)? We have chosen GaN/AlGaN quantum structures because the light emission takes place in the binary material, i.e., in the GaN quantum wells, so we can neglect all alloying effects, which seem to have a significant importance for InGaN.

We have studied the multiquantum well GaN/AlGaN structure grown by MBE in Valbonne [88]. The sample was deposited on the sapphire substrate with the structure described below. First, a few micrometers of undoped GaN were grown, followed by a $0.2 \mu \mathrm{m}$ thick $\mathrm{Al}_{0.17} \mathrm{Ga}_{0.83} \mathrm{~N}$ buffer. Then, a system of $\mathrm{GaN}$ QWs was formed, confined by $100 \AA$ thick $\mathrm{Al}_{0.17} \mathrm{Ga}_{0.83} \mathrm{~N}$ barriers. The QW thicknesses were as follows (counting from the substrate side): 32, 24, 16, 10, 6, 4 monolayers (MLs; $1 \mathrm{ML}=2.59 \AA$ ). Figure 43a illustrates evolution of the photolumi- 

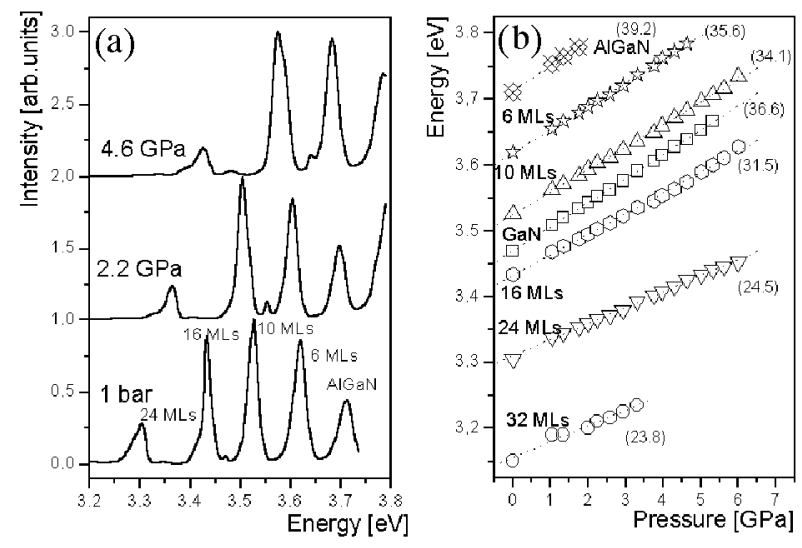

Fig. 43. Photoluminescence of different width of AlGaN/GaN/AlGaN quantum wells in function of the applied pressure: (a) the spectra, (b) pressure coefficient.

nescence spectra with the applied pressure [88]. Figure 43b shows the pressure coefficients of the photoluminescence lines as a function of the QW thickness in the sample studied here.

One can clearly notice that with increasing $\mathrm{QW}$ width the pressure coefficient decreases from about $37 \pm 2 \mathrm{meV} / \mathrm{GPa}$ (for the $6 \mathrm{MLs}$ quantum down to $24 \pm 2 \mathrm{meV} / \mathrm{GPa}$ for the $32 \mathrm{MLs}$ quantum well). Theoretical modeling performed with the purpose of reproducing the experimental results consisting of the PL lines energy values and pressure coefficients of the individual lines shows that the observed $35 \%$ decrease in $\mathrm{d} E / d P$ for 32 monolayers $(8 \mathrm{~nm}$ ) thick quantum well, compared to 6 monolayers well is associated with the increase in the piezoelectric field from $0.66 \mathrm{MV} / \mathrm{cm}$ at ambient pressure to $0.87 \mathrm{MV} / \mathrm{cm}$ at pressure of $10 \mathrm{GPa}$.

In conclusion, we have established that the presence of the electric field in hexagonal InGaN/GaN and GaN/AlGaN quantum structures leads to the drastic reduction of the pressure coefficient of light emission. This effect is related to the increase in the piezoelectric field generated in wurtzite quantum structures of group-III nitrides. To reproduce the experimental findings in InGaN/GaN case, it is necessary to take into account the dependence of the piezoelectric constants on the volume-conserving strain, whereas the experimental results on a decrease in $\mathrm{d} E_{\mathrm{E}} / \mathrm{d} P$ in $\mathrm{GaN} / \mathrm{AlGaN}$ structures can be fully accounted for within the linear elasticity theory.

\section{Optoelectronic devices \\ 6.1. Introduction}

Our pressure grown GaN crystals have been used by Nakamura [89] as substrates for the InGaN MQW-based $405 \mathrm{~nm}$ laser. The $\mathrm{cw} 30 \mathrm{~mW}$ devices with lifetime exceeding $3000 \mathrm{~h}$ were constructed. The $30 \mathrm{~mW}$ power has been achieved at current of $62 \mathrm{~mA}$. For typical InGaN MQW LDs on sapphire [1], at current of 
$62 \mathrm{~mA}$ the output power was then (end of 1999) of about $15 \mathrm{~mW}$ and their lifetime did not exceed $300 \mathrm{~h}$. This increase in the efficiency and lifetime confirmed that dislocations are the main limiting factors for high power GaN based lasers. This results made a final proof that the use of our substrates opens a chance to increase the power of $\mathrm{GaN}$ based laser diodes.

\subsection{Light emitting diodes fabricated on bulk GaN in HPRC}

Basing on the MOCVD growth technique described in Sec. 4, we fabricated a number of light emitting diodes. The example of such diode, obtained in HPRC, is presented in Fig. 44.

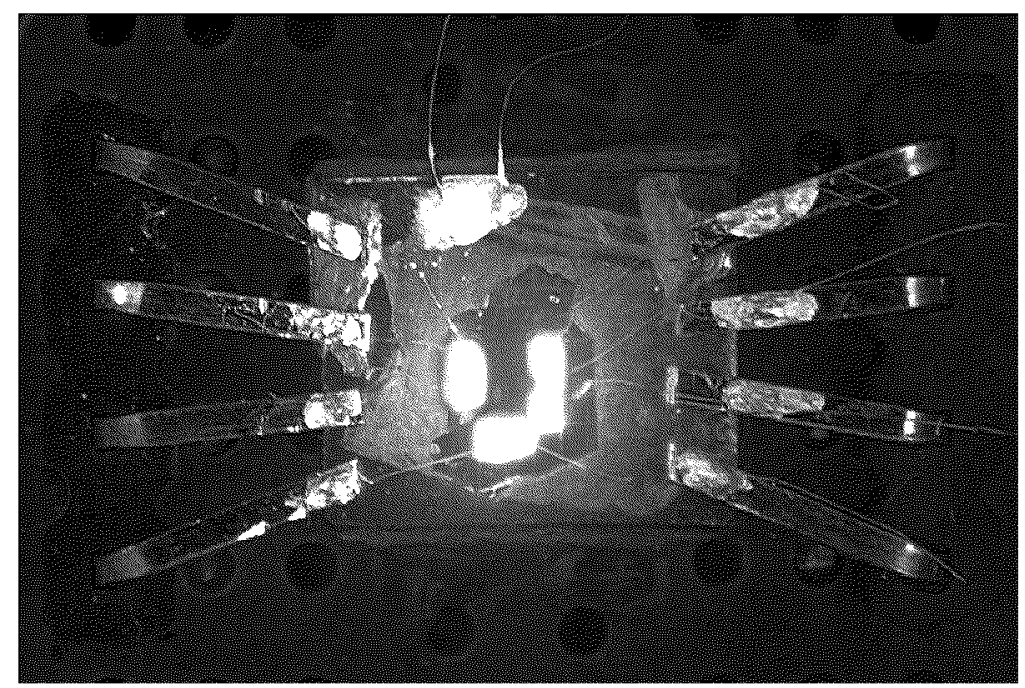

Fig. 44. Blue LED grown on GaN substrate by MOVPE technique.

The typical structure of these devices was the following: first $2 \mu \mathrm{m}$ GaN:Si $\left(n=5 \times 10^{18}\right)$ buffer was deposited on Ga-face of GaN crystal, followed by $10 \times$ multiquantum well $\operatorname{In}_{0.1} \mathrm{Ga}_{0.19} \mathrm{~N} / \mathrm{GaN}(35 \AA / 80 \AA)$. The structure was completed by growing $0.2 \mu \mathrm{m}$ thick GaN:Mg (hole concentration $p=3 \times 10^{17}$ ). The semitransparent $(100 \AA / 50 \AA) \mathrm{Ni} / \mathrm{Au}$ contact was deposited on the top of the structure using e-gun evaporator and annealed in oxygen at $450^{\circ} \mathrm{C}$. Tested devices have dimensions of about $300 \times 300 \mu \mathrm{m}$. Figure $45 \mathrm{a}$ and $\mathrm{b}$ show electrical and optical characteristics of these diodes. It is clearly seen in Fig. 45a that $I-V$ characteristics of our homoepitaxial diode are quite similar to commercially available LEDs produced by Nichia Chemicals. The diodes are characterized by series resistance of about $7-15 \Omega$, very similar to mentioned earlier Nichia devices. However, one should remember that in the case of thin semitransparent contact device series resistance may be seriously influenced by in-plane current spreading. Our metal contact are characterized by contact resistance $(2-5) \times 10^{-3} \Omega \mathrm{cm}^{-2}$. 

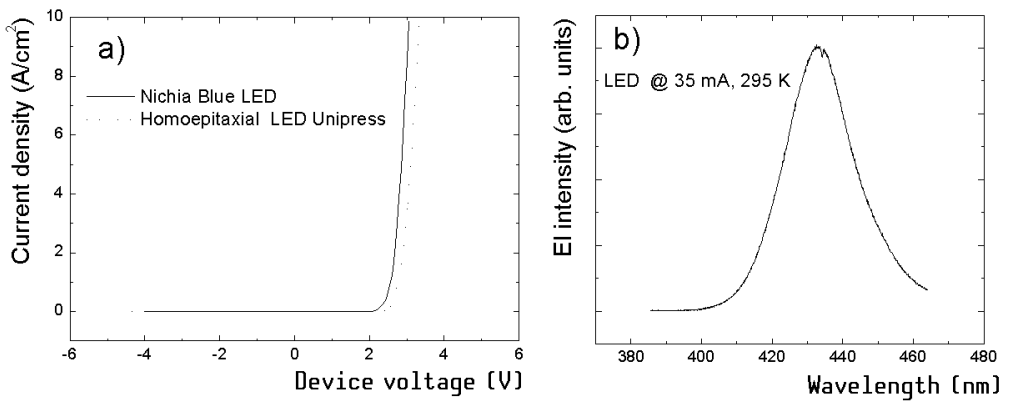

Fig. 45. (a) $I-V$ characteristics of our "blue" LED in comparison with a commercial Nichia device; (b) electroluminescence spectrum of homoepitaxial LED.

\subsection{Laser diode structures}

Our "blue" laser diode design follows this of the classical separate-confinement heterostructure (SCH) laser. Figure 46 shows the details of our structure. The active layer is formed by $\operatorname{In}_{0.1} \mathrm{Ga}_{0.9} \mathrm{~N} / \mathrm{GaN}(30 \AA / 80 \AA) 5-10 \times$ multiquantum well. It is worth noting here that the laser structure is fully strained with no additional dislocation generated. For optical pumping tests, the structures were cleaved to form $500 \mu \mathrm{m}$ long cavity (Fig. 47a). For electrical testing - Ni/Au metal contact was deposited in the form of stripes having width of $10 \mu \mathrm{m}$. In GaN laser the most severe problem is low coefficient of optical reflection. The coefficient can be increased by deposition of dielectric layer $\mathrm{SiO}_{2} / \mathrm{ZrO}_{2}$ (Fig. 47b). As a result, the

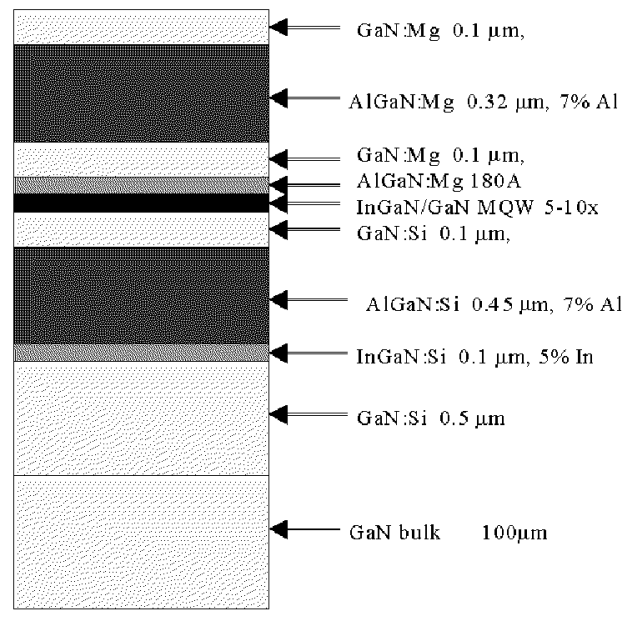

Fig. 46. Design of our "blue" light emitting laser diode structure. 

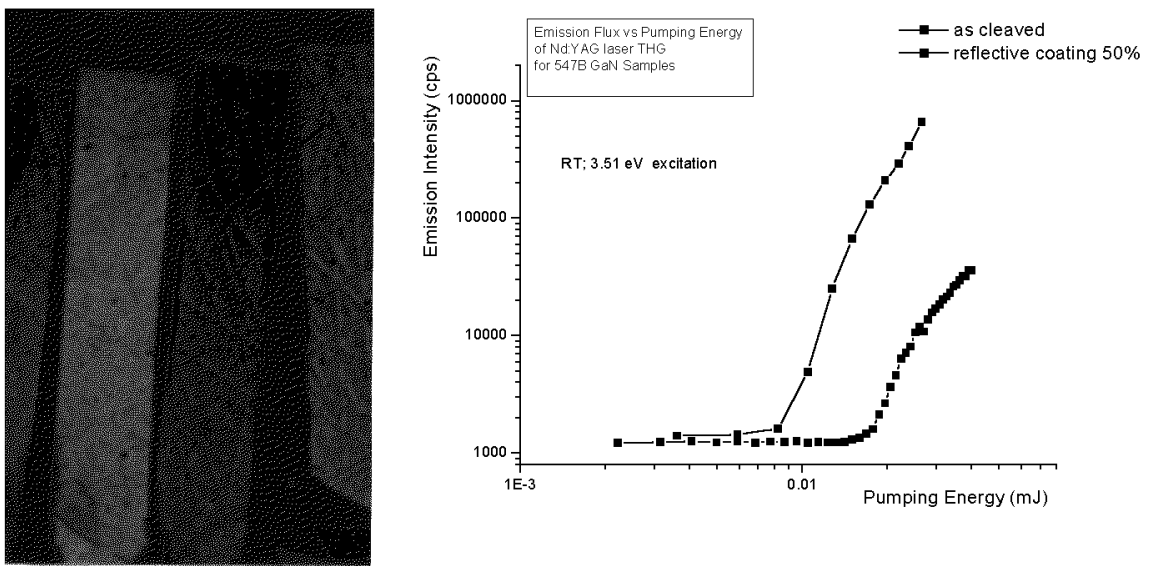

Fig. 47. (a) Laser resonators obtained by cleaving of GaN substrates with the deposited MQW structures, (b) influence of $\mathrm{SiO}_{2} / \mathrm{ZrO}_{2}$ mirror coatings on the laser performance.

coefficient has been increased to $50 \%$. This allowed to decrease the optical injection threshold twice. In the last two years we have successfully demonstrated that homoepitaxially grown GaN epilayers show narrow stimulated emission lines (under optical pumping conditions) at relatively low threshold power densities [90].

The optical pumping of the described above laser structures demonstrated that they are characterized by low optical excitation threshold of about $200 \mathrm{~kW} / \mathrm{cm}^{2}$. The light spectra of our laser are shown in Fig. 48.
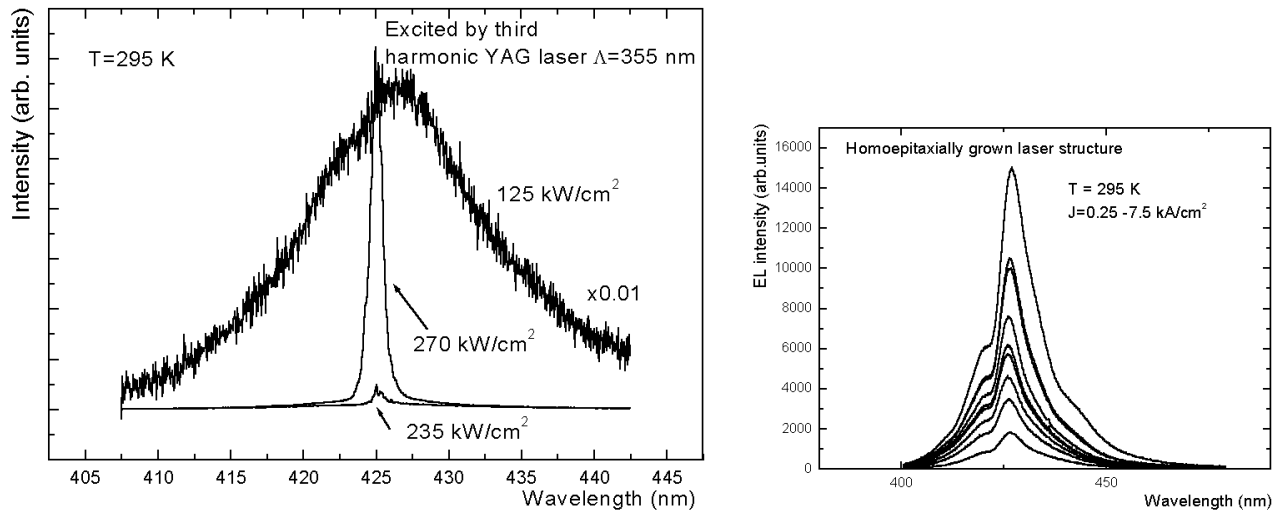

Fig. 48. Emission spectra below and above laser threshold. Spectra measured at room temperature under optical excitation from the third harmonic of YAG laser.

Figure 49 demonstrates the results of the first current injection experiments performed on our structures. The lasing threshold has not been reached so far. The next steps which are being prepared to obtain the laser action include the 

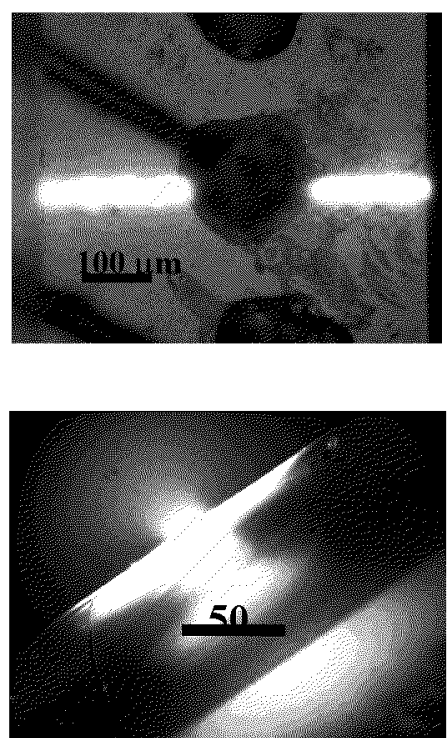

Fig. 49. (a) High-current electroluminescence spectra of homoepitaxial laser structure measured under pulse-current conditions (pulse width $10 \mathrm{~ns}$, repetition - $40 \mathrm{~Hz}$ ), (b) light emission from the laser structure (simplified processing).

improvement of the processing, in particular the optimization of contact geometry, including $\mathrm{SiO}_{2}$ insulated mesas is highly required.

\section{Conclusions}

The extensive studies of thermodynamical properties of $\mathrm{GaN}-\mathrm{Ga}-\mathrm{N}_{2}$ system, especially the experimental evaluation of the $p-T$ three phase equilibrium conditions at large pressure and temperature range, allowed to establish the conditions for crystallization of GaN from solutions of atomic nitrogen in liquid gallium.

The method has been optimized by the experimental studies of growth mechanisms and by quantum mechanical modeling of the interaction of $\mathrm{N}_{2}$ molecules with gallium. At present, GaN crystals almost free of dislocations, with dimensions sufficient to be used as substrates for epitaxy, are grown under pressure. The application of these crystals allows to overcome one of the most important barriers in development of physics and technology of group-III metal nitrides.

The spectacular development of III-V nitrides physics and technology in the last decade, especially the construction of highly efficient blue LEDs and low power LDs, was achieved, although structural quality of the nitride epilayers deposited on highly mismatched substrates was relatively very poor.

The application of GaN substrates grown under pressure leads to the reduction in dislocation density in the quantum $\mathrm{AlGaN} / \mathrm{InGaN}$ structures by $5-6$ orders 
of magnitude. Then the efficiency of the blue laser diodes based on InGaN and the UV emitters based on AlGaN can be significantly increased.

Availability of the near dislocation-free epitaxial layers and structures of nitrides allows also important basic research of physics of these materials since otherwise many physical properties and phenomena are influenced by the presence of structural defects in the crystal lattice.

The advantage of having the near dislocation-free GaN substrates encouraged HPRC to extend its research in nitrides onto the growth and physics of the quantum structures including structures of the blue InGaN based laser. Lasing under optical pumping, at very low threshold power in the InGaN/GaN MQW has been achieved. The processing of the optoelectronic devices is developed up to the point that the full laser epitaxial structures in the separate confinement heterostructure configuration are ready to be tested at high injection current regime.

\section{Acknowledgments}

The research presented in this paper has been supported by the State Committee for Scientific Research grants 8 T11 G 0012000 C/5013 and 7 T08A 01518 and in part by European Commision within the Support for Centers of Excellence No. ICA1-CT-2000-70005. The calculations were made using computing facilities of Interdisciplinary Centre for Mathematical and Computational Modelling of Warsaw University.

\section{Note added in proof}

While this review was in print, we carried out first successful experiment of current-injected laser action (at $425 \mathrm{~nm}$ ) in our "blue" laser diode structure on bulk GaN substrate (described in Sec. 6.3). In the short note (which is also included to this issue, see p. 000), all vital characteristics of the laser diode structure as well as its operational parameters and optical characteristics are reported.

\section{References}

[1] S. Nakamura, G. Fasol, The Blue Laser Diodes, Springer-Verlag, Berlin 1997.

[2] S. Porowski, I. Grzegory, J. Cryst. Growth 178, 174 (1997).

[3] G.A. Slack, T.F. McNelly, J. Cryst. Growth 34, 263 (1976).

[4] J.A. Van Vechten, Phys. Rev. B 7, 1479 (1973).

[5] J. Karpinski, J. Jun, S. Porowski, J. Cryst. Growth 66, 1 (1984).

[6] J. Karpinski, S. Porowski, J. Cryst. Growth 66, 11 (1984).

[7] J.B. Mac Chesney, P.M. Bridenbaugh, P.B. O'Connor, Mater. Res. Bull. 5, 783 (1970).

[8] I. Grzegory, Ph.D. thesis, Warsaw 1995 (in Polish). 
[9] C.D. Thurmond, R.A. Logan, J. Electrochem. Soc. 119, 622 (1972).

[10] R. Madar, G. Jacob, J. Hallais, R. Fruchard, J. Cryst. Growth 31, 197 (1975).

[11] Termodinamicheske svoistva individualnikh veshchestv, Ed. W.P. Glushko, Nauka, Moscow 1979 (in Russian).

[12] W.E. Zinowiev, Teplofizicheske svoistva metallov pri vysokikh temperaturakh, Metallurgia, Moscow 1989 (in Russian).

[13] B. Delley, J. Chem. Phys. 92, 508 (1990).

[14] Z. Romanowski, S. Krukowski, I. Grzegory, S. Porowski, J. Chem. Phys. 114, 6353 (1990).

[15] Z. Romanowski, S. Krukowski, I. Grzegory, S. Porowski, J. Cryst. Growth 189/190, 159 (1998).

[16] J. Neugebauer, C.G. Van de Walle, Phys. Rev. B 50, 8067 (1994).

[17] P. Boguslawski, E.L. Briggs, J. Bernholc, Phys. Rev. B 51, 17255 (1995).

[18] Z. Liliental-Weber, EMIS Datareview Series No. 23, INSPEC, The Institution of Electrical Engineers, London 1999, p. 230.

[19] J.L. Rouviere, J.L. Weyher, M. Seelmann-Eggebert, S. Porowski, Appl. Phys. Lett. 73, 668 (1998).

[20] M. Seelmann-Eggebert, J.L. Weyher, H. Obloh, H. Zimmermann, A. Rar, S. Porowski, J. Cryst. Growth 210, 151 (2000).

[21] W.A. Tiller, K.A. Jackson, J.W. Rutter, B. Chalmers, Acta Metall. 1, 428 (1953).

[22] W.A. Tiller, J. Cryst. Growth 2, 345 (1968).

[23] J.L. Weyher, S. Müller, I. Grzegory, S. Porowski, J. Cryst. Growth 182, 17 (1997).

[24] S. Krukowski, M. Bockowski, B. Lucznik, I. Grzegory, S. Porowski, T. Suski, Z. Romanowski, J. Phys., Condens. Matter 13, 8881 (2001).

[25] P. Perlin, J. Camassel, W. Knap, T. Talercio, J.C. Chervin, T. Suski, I. Grzegory, S. Porowski, Appl. Phys. Lett. 67, 2524 (1995).

[26] E. Litwin-Staszewska, T. Suski, R. Piotrzkowski, I. Grzegory, M. Bockowski, J.L. Robert, L. Konczewicz, D. Wasik, E. Kaminska, D. Cote, B. Clerjaud, J. Appl. Phys. 89, 7960 (2001).

[27] K. Saarinen, T. Laine, S. Kuisma, P. Hautojarvi, L. Dobrzyński, J.M. Baranowski, K. Pakuła, R. Stępniewski, M. Wojdak, A. Wysmołek, T. Suski, M. Leszczyński, I. Grzegory, S. Porowski, Phys. Rev. Lett. 79, 3030 (1997).

[28] S. Porowski, M. Boćkowski, B. Łucznik, I. Grzegory, M. Wróblewski, H. Teisseyre, M. Leszczyński, E. Litwin-Staszewska, T. Suski, P. Trautman, K. Pakuła, J.M. Baranowski, Acta Phys. Pol. A 92, 958 (1997).

[29] T. Suski, E. Litwin-Staszewska, P. Perlin, P. Wiśniewski, H. Teisseyre, I. Grzegory, M. Bockowski, S. Porowski, K. Saarinen, J. Nissila, J. Cryst. Growth 230, 368 (2001).

[30] E. Frayssinet, W. Knap, S. Krukowski, P. Perlin, P. Wisniewski, T. Suski, I. Grzegory, S. Porowski, J. Cryst. Growth 230, 442 (2001).

[31] T. Suski, J. Jun, M. Leszczynski, H. Teisseyre, I. Grzegory, S. Porowski, G. Dollinger, K. Saarinen, T. Laine, J. Nissila, W. Burkhard, W. Kriegseis, B.K. Meyer, Mater. Sci. Eng. B 59, 1 (1998). 
[32] M. Leszczynski, I. Grzegory, H. Teisseyre, T. Suski, M. Bockowski, J. Jun, J.M. Baranowski, S. Porowski, J. Domagala, J. Cryst. Growth 169, 235 (1996).

[33] S.H. Christiansen, M. Albrecht, H.P. Strunk, C.T. Foxon, D. Korakakis, I. Grzegory, S. Porowski, Phys. Status Solidi A 176, 285 (1999).

[34] Z. Liliental-Weber, M. Benamara, W. Swider, J. Washburn, I. Grzegory, S. Porowski, R.D. Dupuis, C.J. Eiting, Physica B 273-274, 124 (1999).

[35] J.L. Weyher, P.D. Brown, J.L. Rouviere, T. Wosinski, A.R.A. Zauner, I. Grzegory, J. Cryst. Growth 210, 151 (2000).

[36] M. Leszczynski, P. Prystawko, G. Nowak, private communication.

[37] M. Leszczynski, P. Prystawko, private communication.

[38] I. Grzegory, Mater. Sci. Eng. B 82, 30 (2001).

[39] See: High Pressure in Semiconductor Physics, Semiconductors and Semimetals, Vols. 54, 55, Eds. T. Suski, W. Paul, Academic Press, San Diego 1998.

[40] P. Perlin, I. Gorczyca, N.E. Christensen, I. Grzegory, H. Teisseyre, T. Suski, Phys. Rev. B 45, 13307 (1992).

[41] W. Shan, J.W. Ager III, W. Walukiewicz, B.D. Little, J.J. Song, M. Shurman, Z.C. Feng, R.A. Stall, B. Goldenberg, Appl. Phys. Lett. 72, 2274 (1998).

[42] T. Suski, Mater. Sci. Forum 143-147, 975 (1994).

[43] P. Perlin, T. Suski, H. Teisseyre, M. Leszczynski, I. Grzegory, J. Jun, S. Porowski, P. Boguslawski, J. Bernholc, J.C. Chervin, A. Polian, T.D. Moustakas, Phys. Rev. Lett. 75, 296 (1995).

[44] J. Neugebauer, C.G. Van de Walle, in: Proc. 22nd Int. Conf. on the Physics of Semiconductors, Vancouver 1994, Ed. D.J. Lockwood, World Sci., Singapore 1995, p. 2327.

[45] C. Wetzel, T. Suski, J.W. Ager III, E.R. Weber, E.E. Haller, S. Fisher, B. Meyer, R. Molnar, P. Perlin, Phys. Rev. Lett. 78, 3923 (1997).

[46] C. Skierbiszewski, T. Suski, P. Wisniewski, W. Jantsch, G. Ostermayer, Z. Wilamowski, P.G. Walker, N.J. Mason, J. Singleton, Appl. Phys. Lett. 63, 3209 (1993).

[47] C.G. Van de Walle, Phys. Rev. B 57, R2033 (1998).

[48] M.T. Hirsch, J.A. Wolk, W. Walukiewicz, E.E. Haller, Appl. Phys. Lett. 71, 1098 (1997).

[49] M.D. McCluskey, N.M. Johnson, C.G. Van de Walle, D.P. Bour, M. Kneissl, W. Walukiewicz, Phys. Rev. Lett. 80, 4008 (1998).

[50] C. Skierbiszewski, T. Suski, M. Leszczynski, M. Shin, M. Skowronski, M.D. Bremser, R.F. Davis, Appl. Phys. Lett. 74, 000 (1999).

[51] M. Stutzmann, O. Ambacher, A. Cros, M.S. Brandt, H. Angerer, R. Dimitrov, N. Reinacher, T. Metzger, R. Hopler, D. Brunner, F. Freudenberg, R. Handschuh, Ch. Deger, Mater. Sci. Eng. B 50, 212 (1997).

[52] A.Y. Polyakov, N.B. Sminnov, A.V. Govorkov, M. Milvidskii, J.M. Redwing, M. Shin, M. Skowronski, D. Greve, R. Wilsons, Solid-State Electron. 42, 627 (1998). 
[53] K. Pakula, A. Wysmolek, K.P. Korona, J.M. Baranowski, R. Stepniewski, I. Grzegory, M. Bockowski, J. Jun, S. Krukowski, M. Wroblewski, S. Porowski, Solid State Commun. 97, 919 (1996).

[54] M. Leszczyński, P. Prystawko, A. Sliwiński, T. Suski, E. Litwin-Staszewska, S. Porowski, R. Paszkiewicz, M. Tłaczała, B. Beaumont, P. Gibart, A. Barski, R. Langer, W. Knap, E. Frayssinet, P. Wiśniewski, Acta Phys. Pol. A 94, 437 (1998).

[55] M. Kamp, C. Kirchner, V. Schvegler, A. Pelzmann, K.J. Ebeling, M. Leszczynski, I. Grzegory, T. Suski, S. Porowski, MRS Internet J. Nitride Semicond. Res. 4S1, G10, 2 (1999).

[56] M. Leszczynski, B. Beaumont, E. Frayssinet, W. Knap, P. Prystawko, T. Suski, I. Grzegory, S. Porowski, Appl. Phys. Lett. 75, 222 (1999).

[57] P. Prystawko, M. Leszczynski, B. Beaumont, P. Gibart, E. Frayssinet, W. Knap, P. Wisniewski, M. Bockowski, T. Suski, S. Porowski, Phys. Status Solidi B 210, 437 (1998).

[58] M. Leszczynski, H. Teisseyre, T. Suski, I. Grzegory, M. Bockowski, J. Jun, S. Porowski, K. Pakula, J.M. Baranowski, C.T. Foxon, T.S. Cheng, Appl. Phys. Lett. 69, 73 (1996).

[59] A. Wysmolek, M. Potemski, R. Stepniewski, J. Lusakowski, K. Pakula, J.M. Baranowski, G. Martinez, P. Wyder, I. Grzegory, S. Porowski, Phys. Status Solidi B 216, 11 (1999).

[60] C. Kirchner, V. Schwegler, F. Eberhard, M. Kamp, K.J. Ebeling, K. Kornitzer, T. Ebner, K. Thonke, R. Sauer, P. Prystawko, M. Leszczynski, I. Grzegory, S. Porowski, Appl. Phys. Lett. 75, 1098 (1999).

[61] A.R.A. Zauner, J.L. Weyher, M. Plomb, V. Kirilyuk, I. Grzegory, W.J.P. Enckevort, J.J. Schermer, P.R. Hageman, P.K. Larsen, J. Cryst. Growth 210, 435 (2000).

[62] S. Jursenas, N. Kurilcik, G. Kurilcik, A. Zukauskas, P. Prystawko, M. Leszczynski, T. Suski, P. Perlin, I. Grzegory, S. Porowski, Appl. Phys. Lett. 78, 3776 (2001).

[63] J.Chr. Holst, A. Hoffman, M. Leszczynski, S. Porowski, private communnication.

[64] M. Leszczynski, P. Prystawko, R. Czernecki, J. Lehnert, T. Suski, P. Perlin, P. Wisniewski, I. Grzegory, G. Nowak, S. Porowski, M. Albrecht, J. Cryst. Growth 231, 352 (2001).

[65] S.H. Christiansen, M. Albrecht, H.P. Strunk, C.T. Foxon, D. Korakakis, I. Grzegory, S. Porowski, Phys. Status Solidi A 176, 285 (1999).

[66] C.T. Foxon, C.S. Davis, S.V. Novikov, O.H. Hughes, T.S. Cheng, D. Korakakis, N.F. Jeffs, I. Grzegory, S. Porowski, Phys. Status Solidi A 176, 723 (1999).

[67] E. Frayssinet, W. Knap, P. Lorenzini, N. Grandjean, J. Massies, C. Skierbiszewski, T. Suski, I. Grzegory, S. Porowski, G. Simin, X. Hu, M.A. Khan, M.S. Shur, R. Gaska, D. Maude, Appl. Phys. Lett. 77, 2551 (2000).

[68] J.D. Lambkin, D.J. Dunstan, K.P. Homewood, L.K. Howard, M.T. Emeny, Appl. Phys. Lett. 57, 1986 (1990).

[69] N. Grandjean, B. Damilano, J. Massies, G. Neu, H. Teisseyre, I. Grzegory, S. Porowski, M. Gallart, P. Lefebvre, B. Gil, M. Albrecht, J. Appl. Phys. 88, $183(2000)$. 
[70] F. Bernardini, V. Fiorentini, D. Vanderbilt, Phys. Rev. B 56, R10024 (1997); V. Fiorentini, F. Bernardini, F. Della Salla, A. Di Carlo, P. Lugli, Phys. Rev. B 60, 8849 (1999).

[71] N. Grandjean, B. Damilano, S. Dalmasso, M. Leroux, M. Laügt, J. Massies, J. Appl. Phys. 86, 3714 (1999).

[72] O. Ambacher, J. Smart, J.R. Shealy, N.G. Weimann, K. Chu, M. Murphy, W.J. Schaff, L.F. Eastman, R. Dmitriev, L. Wittmer, M. Stutzmann, W. Rieger, J. Hilsenbeck, J. Appl. Phys. 85, 3222 (1999).

[73] S. Chichibu, T. Azuhata, T. Sota, S. Nakamura, Appl. Phys. Lett. 69, 4188 (1996).

[74] H. Kollmer, J.S. Im, S. Heppel, J. Off, F. Scholz, A. Hangleiter, Appl. Phys. Lett. 74, 82 (1999).

[75] Yong-Hwan Kwon, G.H. Gainer, S. Bidnyk, Y.H. Cho, J.J. Song, M. Hansen, S.P. DenBaars, Appl. Phys. Lett. 75, 2545 (1999).

[76] S. Chichibu, M. Sugiyama, T. Kuroda, A. Tackeuchi, T. Kitamura, H. Nakanishi, T. Sota, S.P. DenBaars, S. Nakamura, Y. Ishida, H. Okumura, Appl. Phys. Lett., in press.

[77] I. Ho, G.B. Stringfellow, Appl. Phys. Lett. 69, 2701 (1996); G.B. Stringfellow, G.S. Chen, J. Vac. Sci. Technol. B 9, 2182 (1991).

[78] M.K. Behbehani, E.L. Piner, S.X. Liu, N.A. El-Masry, S.M. Bedair, Appl. Phys. Lett. 75, 2201 (1999).

[79] C.A. Parker, J.C. Roberts, S.M. Bedair, M.J. Reed, S.X. Liu, N.A. El-Masry, L.H. Robins, Appl. Phys. Lett. 75, 2566 (1999).

[80] P. Perlin, V. Iota-Herbei, B.A. Weinstein, P. Wisniewski, T. Suski, P.G. Eliseev, M. Osinski, Appl. Phys. Lett. 70, 2993 (1997).

[81] T. Suski, Phys. Status Solidi B, submitted for publication.

[82] P. Perlin, I. Gorczyca, N.E. Christensen, I. Grzegory, H. Teisseyre, T. Suski, Phys. Rev. B 45, 13307 (1992).

[83] N.E. Christensen, I. Gorczyca, Phys. Rev. B 50, 4397 (1994).

[84] S.-H. Wei, A. Zunger, Phys. Rev. B 60, 5404 (1999).

[85] P. Perlin, I. Gorczyca, T. Suski, P. Wisniewski, S. Łepkowski, N.E. Christensen, A. Svane, M. Hansen, S.P. DenBaars, B. Damilano, N. Grandjean, J. Massies, Phys. Rev. B 64, 115319 (2001).

[86] G. Vaschenko, D. Patel, C.S. Menoni, S. Keller, U.K. Mishra, S.P. DenBaars, Appl. Phys. Lett. 78, 640 (2001).

[87] G. Vaschenko, D. Patel, C.S. Menoni, N.F. Gardner, J. Sun, W. Goetz, C.N. Tome, B. Clausen, Phys. Status Solidi B, in press.

[88] S.P. Lepkowski, H. Teisseyre, T. Suski, P. Perlin, N. Grandjean, J. Massies, Appl. Phys. Lett. 79, 1483 (2001).

[89] S. Nakamura, private communication.

[90] P. Perlin, private communication. 